This refort was prepared as an account of wurk sponsofed by an agency of the United Stales Government, Neither the Uijited Stafts Government not any agency thereof, not any of their enployees, makcs any waranty, express or implied, or assumes any legal liability or responsibility for the accuracy, completencss, or usefulness of any inforntation, apparatus, product, or process disclosed, or represents that its use wesuld nut infringe privalelv owned rights. Relerence hereir. !n any specific commetcial product, piuniss, or service by trade name, trademark. munufacturer or otherwise does nol necessarily constitute or inply its endorsement, fecommendation, or favoring by the United Stutes Government of any agency thereof. The views and opinions of euthess sxptessed herein jo not necessarily state or reflect those of the Uniled Stutes Government or any agency thereor.

\title{
A REALISTIC MODEL FOR QUANTUM THEORY WITH A LOCALITY PROPERTY*
}

\author{
Philippe H. Eberhard
}

Lawrence Berkeley Laboratory

University of California

Berkeley, CA 94720

\begin{abstract}
A model seproducing the predictions of relativistic quantum. theory to any desired degree of accuracy is described in this paper. it involves quantities that are independent of the observer's knowledge, and therefore can be called real, and which are defined at each point in space, and therefore can be called local in a rudimentary sense. It involves faster-than-light, but not instantaneous, action at distance.
\end{abstract}

-This work is supported by the U.S. Department of Energy under Contract DE-AC0376 SF00098. 


\section{INTRODUCTION}

A model of reality satisfying a rudimentary property of locality is described here with predictions that can be made to differ from the predictions of relativistic quantum theory by an arbitrary amount. Adjusting a pararister $V$, one can reduce the differences between the model and quantum .theory to an infinitesimal amount, i.e., indistinguishable by experiment. Alternatively, one can adjust $V$ to make some of these differences observable in future more refined experiments and not significant in the previous ones. Within the model, measurement theories can be developed. A framework for them is suggested.

In Section 2, a translation invariant formulation of quantum theory is developed to make it easier to compare its predictions with the predictions of the model. The model is described and compared to quantum theory in Section 3. The properties related to locality are described in Section 4. They are at the origin of some possible experimentally testable discrepancies between the model and quantum theory. Suggestions for an approach to local realistic masurement theories are presented in Section 5.

\subsection{Background and Scope}

Quantum theory is generally considered only as a tool to make predictions, ${ }^{[1]}$ not as a description of the mechanisms behind quantum phenomena. Mathematical entities, such as wave functions and density matrices which are used to define quantum states, depend not only on characteristics of the quantum system itself but on our knowledge of it as well. Mc dels have been sought that would have features that are only characteristic of the system and all disentangled from the mathematical description of our information about it. These features could be called objective reality. In the models proposed so far for more than one particle, ${ }^{[2]}$ reality is described by nonlocal entities that are not simple functions of the coordinates of a point in space and time. They affect measurements performed at many locations in the universe and are modified instantaneously when a measurement is either set up or performed at any one of these locatious. For instance, this is the case of models that use many-body wave functions to describe 
resiity. Thi The picture of reality includes functions of the space coordinates of many points at the same time, not just functions of one point with only one set of space coordinates. Therefore, these models contradict the most rudimentary notion of locality that we would like to attribute to the real world.

The main purpose of this paper is to describe a model of reality that makes predictions nearly indistinguishable from the predictions of relativistic quantum theory without invoking any instantaneous action at distance. The model describes the universe using only mathematical entities that are independent of the observer's knowledge. These entities are functions of a single set of space-time coordinates. Their value influences instantanecusly only quantities defined at the same point and at points infinitesimally close in space. They have the properties expected from a local description of objective reality. As required by Bell's theorem, ${ }^{[4],[3]}$ there are causal effects that propagate at a velocity $V$ larger than the velocity of light, $c$. This is possible because the model assumes the existence of a privileged space-time restframe in which these causal effects always propagate forward in time.

Strictly speaking, the predictions of the model are identical to the predictions of quantum theory only in the limit of the velocity $V$ being infinite. However, taking $V$ to be finite but large enough, it is possible to reproduce the predictions of quantum theory for any experiment that has been performed so far, while using only effects propagating no faster than the finite velocity $V$. The model fits the predictions of quantum theory to any degree of accuracy desired, and it can be said to be local in a rudimentary sense. The concept of locality used here is different from the Lorentz-invariant concepts of locality that led to the EPR paradox ${ }^{[6]}$ because these latter concepts are incompatible with superluminous effects. ${ }^{[4]_{1}[5]}$ I believe this rudimentary concept of locality contains all the locality requirements that could have been expected from a theory before relativity was discovered. The kind of locality aimed at here is the same kind of locality obtained at the time the Maxwell equations were written.

In the model, it is possible to describe measurements as local processes. They occur at one point, are triggered by physical quantities defined at this point, and affect instantaneously only what we call objective reality at this same point. After the measurement process is over at this point, effects responsible for the "collapse of the wave function" propagate in space at 
velocity $V$. This scenario is compatible with the existence of processes acting on quantum systems as measurements do, even if there is no buman around to notice the result.

\subsection{Basic Features of the Model}

In classical physics, the real world is described in terms of particles, which are singularities at some points in space, and of fields, which are scalars, vectors, or other tensoriai quantities with a definite value at each point in space-time. It is not surprising that far more complicated mathematical entities will have to be defined at each point of space-time to describe the complex reality behind quantum theory. For the model described here, the main criterion for choosing one complicater mathematical quantity instead of another for the description of realiity was its ability to make the near equivalence between the model and quantum theory as obvious as possible. Simplisity of the model itself was only the second priority. The goal was to demonstrate the feasibility of models with the rudimentary locality property. It would not be surprising if simplifications of this model or conpietely new simpler approaches could later be found.

The present model makes use of two positive definite hermitian operators in Fock space, $Q(x, t)$ and $C(x, t)$, defined at each point of coordinates $x$ in space and $t$ in time: $Q(x, t)$ will be called the quantum state matrix, with a trace of one, like the trace of a density matrix, and $C(x, t)$ will be called the collapse operator. Though observables, in general, are not localizable in quantum theory, the locality constraint imposed on the model requires that all physical quantities be given a location in space. This requirement applies to observables as well. In the model, an observable $\mathcal{M}$ is attached to a point of measurement $m$ having spatial coordinates $x_{m}$. At the time $t_{m}$ of the measurement, the observable $\mathcal{M}$ becomes manifest at point $m$. This observable $M$ is associated with an operator $M$. The value $\mu$ that $\mathcal{M}$ will have is not determined before measurement, but there is a probability distribution for $M$ that depends only on the operator $M$ and the quantum state matrix $Q\left(x_{m}, t_{m}\right)$ defined locally at point $m$ having coordinates $x_{m}$ and at $t_{m}$.

At time $t_{m}$ of the measurement, the collapse operator $C(x, t)$ is modified 
only at point $m$, i.e. for $x=x_{m}$. Then the change in $C(x, t)$ propagates, away from point $m$, at velocity $V$, according to a linear partial derivative equation in coordinates $r$ and $t$. The collapse operator $C(x, t)$ for each set of values of $x$ and $t$ plays a role in the time evolution of the quantum state matrix $\underline{Q}(x, t)$ defined for the same $x$ and $t$. It malses $\underline{Q}(x, t)$ collapse appropriately to reproduce the predictions of quantum theory for future measurements.

Whether or not measurements are performed, the evolution of $\underline{Q}(x, t)$ and $C(x, t)$ at a point of coordinates $x$ never depends instantaneously on the values of any operator $Q\left(x^{\prime}, t\right)$ or $C\left(x^{\prime}, t\right)$ defined at a distant point having coordinates $x^{\prime}$, nor does it depend on any operator $M$ or any observable associated with a measurement occuring at coordinates $x_{m}$ distant from $x$. The scenario sketched here obviously satisfies the rudimentary locality property wanted. Sections 2 and 3 will demonstrate how such a scenario can be made to produce predictions nearly identical to those of quantum theory. In Section 5, ideas will be given as to how a measurement theory can be developed in this context.

\subsection{Possible Experimental Evidence}

For any finite value of the parameter $V$, there are large distances $\Delta x$ and small time intervals $\Delta t$ such that

$$
\Delta t<\frac{|\Delta x|}{V}
$$

Suppose two measurements are performed at a distance $\Delta x$ and a time interval $\Delta t$ satisfying inequality (1.1). The propagation of the collapse operator $\mathcal{C}(x, t)$ is too slow for the first of these measurements to affect the second one before that second one is performed. Quantum theory assumes instantaneous collapse at any distance. Therefore, for measurements spaced by such $\Delta x$ and $\Delta t$, differences between the model and quantum theory predictions may be expected. This idea will be discussed in Section 4.

In principle, these differences between model and quantum theory can be looked for experimentally. The model provides a basis for testing quantum theory experimentally in a direction where quantum theory may sometimes be suspected to fail if a model of this type is correct. If a violation is 
found, it may corroborate the ideas behind this model. However, whenever quantum theory predictions are upheld, experiment does not rule out the model. It only establishes a lower limit for parameter $V$.

It should be pointed out that, whether or not such a violation of quantum theory predictions is found experimentally, the model can always be used by those physicists and engineers who prefer to work with real localizable entities rather than with the present orthodox concepts of quantum theory. In particular, the ideas presented in Section 5 for possible realistic descriptions of the measurement process could be used regardless of the result of experiments trying to determine the value of $V$.

\section{FIELD THEORY AND TRANSLATION-INVARIANT OPERATORS}

In this section, orthodox quantum theory is given an unusual formulation that later will be easier to compare with the model. Our purpose is to express the properties of relativistic quantum theory using, as much as possible, the same operators as the ones to be used in the model. We develop this unusual formulation from a more conventional one using the Schroedinger representation, where state vectors are time dependent and measurement operators are not. To simplify the equations, we use a system of units where $\hbar=1$. In the appendices, some computations are simplified by using operators defined in the Heisenberg representation.

\subsection{The Density Matrices for the Universe and for an Isolated Quantum System}

We will make use of operators defined for relativistic quantum field theory in the space called Fock space, ${ }^{[7]}$ In this space, the elements are sets of functions of momentum $p$. The wave function of a system of an undetermined number of particles is an element of Fock space. An element of Fock space, $\Psi$, contains a zeroth order function that is a complex number $\psi_{0}$. In the wave function interpretation, $\psi_{0}$ is the probability amplitude of the vacuum state. The first-order function is a complex function $\psi_{1, j}(p)$ of 
momentum $p$ and of an index $j$ that corresponds to a field and, for a multicomponent field, to a given component of that field. In the wave function interpretation, $\psi_{1, j}(p)$ is the probability amplitude of having one particle in the system, in the field defined by $j$, with the component determined by that same index $j$ and having momentum $p$. The second-order function is a function $\psi_{2, j_{1}, j_{2}}\left(p_{1}, p_{2}\right)$ of the momenta $p_{1}$ and $p_{2}$, as well as of the indices $j_{1}$ and $j_{2}$, corsesponding to a state with two particles. The $n^{\text {th }}$ order function is a function $\psi_{n, j_{1} \ldots j_{n}}\left(p_{1} \ldots p_{n}\right)$ of $n$ momenta $p_{1} \ldots p_{n}$ and $n$ indices $j_{1} \ldots j_{n}$, corresponding to a state with $n$ particles. The functions $\psi_{n}$ 's have symmetry and antisymmetry properties to satisfy Bose and Fermi statistics, whichever are relevant. Fock space is also the space in which we will define the operators necessary for our model of reality.

One can use the elements of Fock space to define wave functions $\Psi_{u}$ for the system of all the particles of the universe. There are many possible such wave functions because of all the uncertainties that affect our knowledge of most particles in the universe. Using the index $k$ to label these possible wave functions $\Psi_{u, k}$ of the universe, our knowledge of the universe can, at best, associate a probability $w_{k}$ to a possible wave function $\Psi_{u, k}$. The desisity matrix of the universe is defined as

$$
D_{\mathrm{u}}=\sum_{k} w_{k} \Psi_{\mathrm{u}_{k},} \Psi_{\mathrm{u}, k}^{+}
$$

where $\Psi_{u, k}^{+}$is the hermitian adjoint of the element of Fock space $\Psi_{u, k}$.

The matrix $D_{u}$ of the universe is a convenient extrapolation of the concept of density matrix for a system of a limited number of particles. It will be used to define convenient quantities in the comparison of the model to quantum theory. However, all practical applications of quantum theory, i.e., all the cases where a comparison with the model is meaningful, concern systems $S$ with fewer variables than all the variables of the universe. The density matrix of system $S$ is related to the matrix $D_{4}$ by the following equation

$$
D_{S}=\operatorname{Tr}_{\operatorname{ex}(S)} D_{v}
$$

where the partial trace is taken over all variables $\operatorname{ext}(S)$ not belonging to $S$. In certain cases, $D_{S}$ can be approximated by a matrix of rank 1 . Then 
a system wave function $\Psi_{S}$ of the system variables can be defined.

$$
D_{S} \simeq \Psi_{S} \Psi_{S}^{+}
$$

However, we will consider the general case where Eq. (2.3) is not necessarily valid, and we will express our knowledge of a system in quantum theory by its density matrix.

\subsection{Measurement Probabilities and Collapses}

In the practical applications of quantum theory, measurements are represented by operators $M_{S}$ in the space of the system variables $S$. There is a probability $\mathcal{P}_{\mu}$ for the outcome $\mu$ of a measurement of the observable $\mathcal{M}$. Then, the state of the system is changed because, according to conventional quantum theory, a measurement is also a preparation of the system for fitture measurements. The density matrix $D_{S}$ is a function of time $D_{S}(t)$. Let $t_{m}$ be the time of the measurement and $e$ an infinitesimal quantity. Then $\mathcal{P}_{\mu}$ can be computed from the density matrix $D_{S}\left(t_{m}-t\right)$ using a projection operator $\Pi_{S_{\mu}}$ in the space of the system variables $S$.

$$
\mathcal{P}_{\mu}=\operatorname{Tr}\left[\Pi_{S \mu} D_{S}\left(t_{m}-\epsilon\right)\right]
$$

After the measurement has been made and the outcome has been found to be a value $\mu$, the density matrix in the system variables undergoes a change called "collapse"

$$
D_{S}\left(t_{m}+\epsilon\right)=\frac{1}{\mathcal{P}_{\mu}} \Pi_{S_{\mu}} D_{S}\left(t_{m}-\epsilon\right) \Pi_{S_{\mu}}
$$

To some extent it is arbitrary to choose which variables should be kept as system variables and which ones can be eliminated in the partial trace operation of Eq. (2.2). It was pointed out that apparatus variaibles in particular can be considered either way, ${ }^{[8]}$ but the boundaries of the apparatus are uncertain. When we compare the predictions of our model of reality with the ones of conventional quantum theory, we want to bypass the discussion as to which variables are system variables and which ones are external to the system. This can be done by defining the measurement 
operator $M$ and the projection operators $\Pi_{\mu}$ in the original Fock space with the variables of all the particles of the universs. First, we include the apparatus variables in the system $S$ and then complete the set of variables using the identity operator $I_{\text {ext }(S)}$ in the space of the variables ext $(S)$ that are external to the combined system, i.e., of the variables that are not affected by the measurement.

$$
\Pi_{\mu}=\Pi_{S_{\mu}} \times I_{\text {ext(S) }}
$$

Then taking into account Eq. (2.2), Eq. (2.4) can be rewritten for the probability $\mathcal{P}_{\mu}$.

$$
\left.\mathcal{P}_{\mu}=\eta \mathrm{r}\left[\Pi_{\mu} \Gamma{ }_{,} i_{\pi}-\epsilon\right)\right]
$$

The density matrix $D_{u}$ of the universe is a function of time $D_{u}(t)$, submitted to collapses, like $D_{S}(t)$ in Eq. (2.5).

$$
D_{u}\left(t_{m}+\epsilon\right)=\frac{1}{\mathcal{P}_{\mu}} \Pi_{\mu} D_{u}\left(t_{m}-\epsilon\right) \Pi_{\mu}
$$

Now the uncertainty about which variables are system variables and which ones are not is buried in the definition of the operators $\Pi_{\mu}$. Further discussion of this point is postponed until Section 5 after the formal comparison between model and quantum theory has been made.

\subsection{Translation Invariant Operators}

In Fock space, one defines annihilation and creation operators $a_{j}(p)$ and $a_{j}^{+}(p)$ of particles of momentum $p$ and of mass plus kinetic energy $E_{j}(p)$ in the field and with a field component defined by $j$. Using the Fourier transforms of $a_{j}(p)$ and $a_{j}^{+}(p)$, one defines field operators $\varphi_{j}(x)$ at each point of space of coordinates $x$. The general form of $\varphi_{j}(x)$ is ${ }^{[7]}$

$$
\varphi_{j}(x)=\int \sum_{j^{\prime}} g_{j j^{\prime}} a_{j^{\prime}}(p) e^{i p x} \frac{d p^{3}}{E_{j}(p)}+\int \sum_{j^{\prime}} g_{j j^{\prime}}^{\prime} a_{j^{\prime}}^{+}(p) e^{-i p x} \frac{d p^{3}}{E_{j}(p)}
$$

Where the coefficients $g_{j j^{\prime}}$ and $g_{j j^{\prime}}^{\prime}$ depend not only on $p$ but also on the field and field components involved and referred to by the indices $j$ and $j^{\prime}$. Consider a given point $X$ having spatial coordinates $x$ in some restframe. 
The same point $X$ has different coordinates $x^{t}$ in a different restframe having a different origin of the space coordinates. Therefore, the field at this point $X$ corresponds to a different operator $\varphi_{j}\left(x^{\prime}\right) \neq \varphi_{j}(x)$ when the origin is changed.

Let $L_{\pi}$ be the size of a measuring apparatus. The corresponding measurement operator $M$ and projection operators $\Pi_{\mu}$ are functions of weighted averages $\phi_{j}$ of operators defined in a finite region of space

$$
\begin{aligned}
M & =M\left(\phi_{j}\right) \\
\Pi_{\mu} & =\Pi_{\mu}\left(\phi_{j}\right)
\end{aligned}
$$

where all the $\phi_{j}$ 's are of the form

$$
\phi_{j}=\int f_{j}(x) F_{j}\left[\varphi_{j}(x)\right] d x^{3}
$$

where the term $F_{j}\left[\varphi_{j^{\prime}}(x)\right]$ under the integral sign stands for any function of the $\varphi_{j}$ 's of Eq. (2.9) and of their derivatives. The finctions $f_{j}(x)$ of space coordinates $x$ have a support of size $L_{m}$ localized at the apparatus position and hence depend upon the location of the origin of the space coordinates. It follows that the operators $M$ and $\Pi_{\mu}$ have an expression that depends on the choice of origin of the space coordinates.

To make the model translation invariant, we will want operators that do not depend on the choice of coordinates' origin. In quantum theory, a measurement operator with such a property can be defined by choosing an arbitrary point $m$ of coordinates $x_{m}$ inside the apparatus and using the total momentum operator $P$, which, in Fock space, is defined as

$$
P=\sum_{j} \int \frac{d p^{3}}{E_{j}(p)} p a_{j}^{+}(p) a_{j}(p)
$$

Given a vector $\Delta x$ representing a displacement in space, $P$ has the property that

$$
e^{-i P \Delta x} \varphi_{j}(x) e^{i P \Delta x}=\varphi_{j}(x+\Delta y)
$$

Therefore, a relation exists between the measurement operators, $M_{1}$ and $M_{2}$, associated with two identical measuring apparati located at different positions defined by the coordinates $x_{m 1}$ and $x_{m 2}$

$$
M_{2}=e^{-i P\left(x_{m 2}-x_{m l}\right)} M_{1} e^{i P\left(x_{m 2}-x_{m 2}\right)}
$$


with a similar relation for the $\Pi_{t:}$ 's.

These properties allow us to define a measurement operator $\underline{M}$ and projection operators $\underline{\Pi}_{\mu}$ that are characteristic of a measuring apparatus and are independest of the distance $x_{m}$ between the apparatus and the origin of the space coordinates. The operators $\underline{M}$ and $\underline{I I}_{\mu}$ are equal to $M$ and $\Pi_{\mu}$ corresponding to the sarre measuring apparatus but located at a position such as $x_{m}=0$, i.e., at the origin of the space coordinates.

$$
\begin{aligned}
& \underline{M}=e^{i P x_{m}} M e^{-i P x_{m}} \\
& \underline{\Pi}_{\mu}=e^{i P x_{m}} \Pi_{\mu} e^{-i P x_{m}}
\end{aligned}
$$

If a different point $m$ is chosen inside the apparatus to represent its location, the operators $\underline{M}$ anc $\underline{\Pi}_{\mu}$ are somewhat different. But $\underline{M}$ and $\underline{\Pi}_{\mu}$ are independent of the choice of origin of space coordinates, and that is what is important now. How point $m$ should be chosen is a subject for measurement theory which, will be discussed in Section 5 .

Note that if $M_{1}$ and $M_{2}$ refer to identical measuring apparati at different locations in space, then Eq. (2.15) makes it obvious that $\underline{M}_{2}$ and $\underline{M}_{2}$ are the same. Operator $\underline{M}$ is also translation invariant in this sense.

\subsection{The Translation-Invariant Formalism}

Iu make the future comparison between the model and quantum theory easier, we construct a quantum-theory formalism using only transiationinvariant operators. Using the operators $\underline{I}_{\mu}$ : Eq. (2.7) can be rewritten

$$
\mathcal{P}_{\mu}=\operatorname{Tr}\left[\underline{\Pi}_{\mu} D\left(x_{m}, t_{m}-\epsilon\right)\right]
$$

where

$$
D(x, t)=e^{i P x} D_{y}(t) e^{-i P z}
$$

The matrix $D\left(x_{m}, t\right)$ is the density matrix one would have if the origin of the space coordinates had been defined at the point of measurement $m$. It is dependent on point $m$, where the measurement is performed, bui independent of the choice of origin of space coordinates. $D_{u}$ has just the inverse properties. Determining $\mathcal{P}_{\mu}$ from Eq. (2.18) makes use of quantitics 
that are translation invariant, i.e., independent of the choice of origin of space coordinates.

When a measurement is performed, and the density matrix $D_{u}(t)$ collapses according to Eq. (2.8), the $x$-dependent density matrix $D(x, t)$ defined by Eq. (2.19) collapses instantaneously, for all $x$ 's, at time $t_{m}$ of the measurement.

$$
\underline{D}\left(\pi, t_{m}+\epsilon\right)=\frac{1}{\mathcal{P}_{\mu}} \underline{\pi}_{\mu}(x) \underline{D}\left(x, t_{m}-\epsilon\right) \underline{\pi}_{\mu}(x)
$$

where $\pi_{\mu}(x)$ is another projection operator independent of the origin of the space coordinates. The times are defined in the space-time restframe chosen to make our calculations.

$$
\underline{\pi}_{\mu}(x)=e^{i P\left(x-x_{m}\right)} \underline{\Pi}_{\mu} e^{-i P\left(x-x_{m}\right)}=e^{i P_{x}} \Pi_{\mu} e^{-i P x}
$$

Between measurements, the original density matrix $D_{u}$ obeys an equation involving the Hamiltonian operator $H$, which, in Fock space, is defined as

$$
H=\sum_{j} \int \frac{d p^{3}}{E_{j}(p)} E_{j}(p) a_{j}^{+}(p) a_{j}(p)+\text { interaction energy }
$$

where $E_{j}(p)$ is the sum of the mass and kinetic energy of a particle of momentum $p$ in the field defined by index $j$ as in Eq. (2.9). Operator $H$ in this paper is assumed to be constant in time and to commute with $P$

$$
H P-P H=0
$$

Between measurements, the matrix $D_{u}(t)$ evolves in time according to the equation

$$
\frac{d D_{\mathrm{u}}(t)}{d t}=-i\left(H D_{\mathrm{u}}(t)-D_{\mathrm{u}}(t) H\right)
$$

From the definition (2.19) of $\underline{D}(x, t)$, we get its time evolution using Eqs. (2.24) and (2.23).

$$
\frac{\partial \underline{D}(x, t)}{\partial t}=-i[H \underline{D}(x, t)-\underline{D}(x, t) H]
$$

Another condition can be deduced from the definition (2.19) of $\underline{D}(x, t)$. We call this condition the spatial-compatibility condition. Given any set of two points of coordinates $x_{1}$ and $x_{2}$, 


$$
\underline{D}\left(x_{2}, t\right)=e^{i P\left(x_{2}-x_{1}\right)} \underline{D}\left(x_{1}, t\right) e^{-i P\left(x_{2}-x_{1}\right)}
$$

To determine the probabilities $\mathcal{P}_{\mu}$ predicted by quantum theory, a usual formalism consists of using Eq. (2.7) with an adequate definition of the measurement associated operator $\Pi_{\mu}$. The time evolution of the density matrix $D_{u}(t)$ is determined by Eq. $(2.24)$ between measurements using a proper definition of operator $H$ and by Eq. (2.8) at the time of the measurement. The initial value of $D_{u}(t)$ is obtained from the outcome of previous measurements and, possibly, from some preconceived ideas one might have about the universe.

It is more convenient to compare our model's predictions to another formalism that we call the translation-invariant formalism and that makes use only of guantities that do not depend on the choice of origin of the space coordinates. The probabilities $\mathcal{P}_{\mu}$ are obtained via Eq. (2.18) from the operators $\underline{\Pi}_{\mu}$ defined by Eq. (2.17) and $\underline{D}(x, t)$ related to $D_{u}(t)$ by Eq. (2.19). This formalism does not require the definition of $D_{u}(t)$ if the matrix $\underline{D}(x, t)$ is calculated using the equations ruling its evolution, i.e., Eq. (2.25) between measurements and Eq. (2.20) at the time of measurement. To the initial conditions for $\underline{Q}(x, t)$ derived from outcomes of previous zeesurements and to the ones due to preconceived ideas about the universe one has now to add Eq. (2.26) as another constraint. It is enough to introduce this spatialcompatibility condition as an initial condition only because, if $H$ and $P$ commute as assumed in Eq. (2.23) and if Eq. (2.26) is valid at some time $t=t_{0}$, the condition (2.26) will be valid at any later time $t>t_{0}$. This statement is, indeed, true both when the evolution of $\underline{D}(x, t)$ is ru! : $y$ Eq. (2.20) and when it obeys Eq. (2.25).

We will use the translation-invariant formalism to compare the predictions of quantum theory with the ones of the model.

\subsection{Lorentz Invariance}

Either the standard formalism or the translation-invariant formalism described above requires the choice of a space-time restframe in which the matrix $D_{4}(t)$ or $\underline{D}(x, t)$ evolves according to Eqs. (2.24) or (2.25) most of the time and collapses according to Eqs. (2.8) or (2.20) when a mea- 
surement is performed. The sequence of collapses matches the time order of the measurements in the chosen space-time restframe. When measurements are performed at different points in space outside of the light cone of one another, that time order depends on the restframe chosen. In this sense, these formalisms are not Lorentz invariant. However, tensorial covariance and commutation or anticommutation properties are given to the field operators used in the definition of the Hamiltonian $H$ and of the riêasurement operators so that some invariance is preserved in the theory. At the end of the computation the probability $\mathcal{P}_{\mu}$ of the outcome $\mu$ of any measurement of an observable $\mathcal{M}$ is independent of the space-time restframe chosen, once the covariance of $\mathcal{M}$ is properly taken into account. In this sense, the predictions for the observables are Lorentz invariant.

In model of reality where objective reality is given to some of the mathematical quantities used in a non-Lorentz invariant formalism, the description of reality may also not be Lorentz invariant. Our model will have a privileged space-time restframe in which equations are a little simpler than in others. Since the choice of restframe in relativistic quantum theory is arbitrary, we may as well choose that privileged restframe to make our comparison. With a proper adjustment of the model's parameters, we will demonstrate the approximate equivalence of the computations of probabilities in the model and in quantum theory when the quantura theory calculations are made in the privileged restframe. Since one cer choose any restframe for computation in quantum theory and find the same probability distributions, one can also assume any restframe to be the privileged one in the model, and computations will also yield the same probabilities. The probabilities computed in the model have the same Lorentz invariance with respect to the choice of privileged restrame as the quantum-theory probabilities with respect to the choice of restframe to make computations.

In the model, as in quantum theory, observers have access only to information contained in the observables. Insofar as the model parameters are adjusted to generate probabilities independent of the choice of restframe assumed to be the privileged one, all observable predictions will be independent of that choice. Therefore, measurement results will not allow an observer to determine which one of the restframes is the privileged one.

Of course, this Lorentz invariance of the model's predictions holds only for those values of the parameters that make the model equivalent to rela- 
tivistic quantum theory. This invariance can be expected to be broken for other values of the parameters.

\section{THE MODEL AND ITS PREDICTIONS}

In the model, as in quantum theory, there are observables $\mathcal{M}$. These observables become manifest in measuring apparati around points $m$ of coordinates $x_{m}$ chosen to define the location of the apparati. In the model, the observable $\mathcal{M}$ is considered as a quantity attached to point $m$. Measurement theory is supposed to provide a relation hetween each apparatus and an operator $\underline{M}$ that specifies the characterisitics of the measurement process. The operator $\underline{M}$ is the same translation-invariant operator as the operator defined by Eq. (2.16) for quantum theory. For the model it is a quantity describing a measurement process occurring at point $m$ at time $t_{m}$.

Reality in the model is further described by two other operators, the "quantum-state matrix" $Q(x, t)$ and the "collapse operator" $C(x, t)$ defined at each point of coordinate $x$ of space at any time t. All predictions require only operators $M, Q$, and $C$. All these operators are defined as local quantities and translation invariant. In this section, we will write equations for them and set parameters to make the model reproduce the predictions of quantum theory to any degree of accuracy desired.

\subsection{Probabilities of Measurement Results}

In the model, the characteristics of the measuring apparatus are expressed by operator $\underline{M}$ of Eq. (2.16) instead of $M$ of Eq. (2.10). Because $M$ is defined as an operator independent of the choice of origin of the space coordinates, it is the reasonable quantity to use in a translation-invariant model. There are several possible results to a measurement with a probability associated to each of them. These possible results are the eigenvalues $\mu$ of $\underline{M}$, which are the same as the eigenvalues of $M$ as can easily be shown using Eq. (2.16), and are in agreement with the rules of quantum theory. Each eigenvalue $\mu$ corresponds to a projection operator $\prod_{\mu}$ that transforms 
any element of Fock space into an eigenvector of $\underline{M}$ with eigenvalue $\mu$.

Operator $Q(x, t)$ is a positive definite matrix of trace one. When an apparatus characterized by operator $M$ performs a measurement at point $m$ having spatial coordinates $x_{m}$ at time $t_{m}$, the probability of outcome $\mu$ is

$$
\wp_{\mu}=\operatorname{Tr}\left[\underline{\Pi}_{\mu} \underline{Q}\left(x_{m}, t_{m}\right)\right]
$$

The quantum matrix $Q(x, t)$ contains all the information that could impact on all possible measurements one could perform at the point of coordinates $x$ at time $t$, and it may even contain more. One cannot expect to have all this information available to us with $100 \%$ accuracy when we make a prediction about a measurement. Therefore, for any prediction, it is necessary to consider several possible quantum matrices $\underline{Q}_{k}(x, t)$ with weights $w_{k}$ to take into account our uncertainty about the real quantum matrix $Q(x, t)$. The weights $w_{k}$ 's are not part of the description of objective reality. Each $w_{k}$ is the probability we attribute to the likelihood that this real quantum matrix actually is $\underline{Q}_{k}(x, t)$. There is a conditional probability, $\wp_{k \mu}$, that the apparatis measures the result $\mu$ when the quantum matrix is $\underline{Q}_{k}(x, t)$, and, given our uncertainty about $\underline{Q}$, the probability $\mathcal{P}_{\mu}$ of the outcome is

$$
\mathcal{P}_{\mu}=\sum_{k} w_{k} p_{k \mu}=\operatorname{Tr}\left[\underline{\Pi}_{\mu} \underline{D}\left(x_{m}, t_{m}\right)\right]
$$

making use of a matrix $\underline{D}(x, t)$ which is also not part of the description of reality and is defined for all $x$ and $t$,

$$
\underline{D}(x, t)=\sum_{k} w_{k} \underline{Q}_{k}(x, t)
$$

In our model, the probability $\mathcal{P}_{\mu}$ of observing the outcome $\mu$ is related to a matrix $\underline{D}(x, t)$ via Eq. (3.2) as in quantum theory in the translationinvariant formalism of Section 2. Therefore, we call $\underline{D}(x, t)$ the translationinvariant density matrix of the model. Equation (3.2) is equivalent to Eq. (2.18) because, in the madel, the time evolution of $Q(x, t)$, thus of $D(x, t)$ until new information about the $w_{k}$ 's reaches the observer, will basically be continuous. When these evolutions appear to be discontinuous, it will be because of the extremely short duration $\tau_{m}$ of a transition period following $t_{m}$. 
The model and quantun theory will have approximately the same predictions if their density matrices $D(x, t)$ are approximately the same. Throughout Section 3 we will write equations for the evolution of $Q(x, t)$ so that $\underline{D}(x, t)$ behaves as in quantum theory as much as possible. As pointed out in Section 2.5, it will be enough to show the approximate equivalence between quantum theory and model in only one space-time restframe since the choice of the restframe in quantum theory is completely arbitrary.

\subsection{Equations for Time Evolution}

In one restframe, which we choose to make our comparisou with quantum theory, the matrix $\underline{Q}(x, t)$ satisfies the following equation

$$
\begin{aligned}
\frac{\partial \underline{Q}(x, t)}{\partial t} & =-i[H \underline{Q}(x, \dot{t})-\underline{Q}(x, t) H]+\underline{C}(x, t) \underline{Q}(x, t)+\underline{Q}(x, t) \underline{C}(x, t) \\
& -2 \operatorname{Tr}[\underline{C}(x, t) \underline{Q}(x, t)] \underline{Q}(x, t)
\end{aligned}
$$

where $C(x, t)$ is one of the above-mentioned Fock-space operators needed to describe reality at point $X$ of coordinates $x$ at time $t ; C(x, t)$ is called the collapse operator. It is a positive definite matrix. It is zero most of the time and, after a measurement is made at time $t_{m}$ at point $m$ having coordinates $x_{m}, \underline{C}(x, t)$ takes non-zero values for a short period of duration $\tau_{m}$ between time $t_{i}=t_{m}+T(x)$ and time $t_{f}=t_{m}+T(x)+\tau_{m}$. That period will be called the transition period. The delay $T(x)$ between time $t_{m}$ of the measurement and time $t_{i}$ of the beginning of the transition period is proportional to the distance $\left|x-x_{m}\right|$ between point $m$ and point $X$ of coordinates $x$ under consideration.

$$
T(x)=\frac{\left|x-x_{m}\right|}{V}
$$

where $V$ is a parameter of the model; $V$ is the velocity at which the zone where $C(x, t)$ is nonzero propagates away from the point of measurement $m$. The duration $\tau_{m}$ of the transition period is a parameter characteristic of the measurement of $\mathcal{M}$. There is also another parameter, $\lambda_{m}$.

$$
\underline{C}(x, t)=\lambda_{m} \underline{\pi}(x, t)\left[\Theta\left(t-T(x)-t_{m}\right)-\Theta\left(t-T(x)-\tau_{m}-t_{m}\right)\right]
$$


where $\Theta(t)$ is the Heaviside function and $\pi(x, t)$ a projection operator related to projection operator $\underline{\pi}_{\mu}(x)$ of Eq. (2.21), thus $\underline{\Pi}_{\mu}$ of Eq. (2.17),

$\underline{\pi}(x, t)=e^{-i H\left(t-t_{m}\right)} \underline{\pi}_{\mu}(x) e^{i H\left(t-t_{m}\right)}=e^{-i H\left(t-t_{m}\right)} e^{i P\left(x-x_{m}\right)} \underline{\Pi}_{\mu} e^{-i P\left(x-x_{m}\right)} e^{i H\left(t-t_{m}\right)}$

In Appendix $A$ it is shown that operator $C(x, t)$ of Eq. (3.6) is just the solution of a linear partial derivative equation in $x$ and $t$ labeled Eq. (A.17). This squation is a linear equation for $C(x, t)$. If several measurement processes are occurring at different points, the collapse operator $\underline{C}(x, t)$ will still be assumed to obey that same linear equation. Therefore, $C(x, t)$ will be the sum of the contributions given by Eq. (3.6) of each individual measurement.

A period during which collapse operator $C(x, t)$ is zero will be called a transition-free period. During such a period Eq. (3.4) reduces to

$$
\frac{\partial \underline{Q}}{\partial t}=-i[H \underline{Q}(x, t)-\underline{Q}(x, t) H]
$$

When we want to minimize the differences between the predictions of the model and the ones of quantum theory, we consider only extremely large values of velocity $V$. We also give very small values to parameter $\tau_{m}$. The time delay $T(x)$ of Eq. (3.5) is very small for all values of $x$. The transition period is of short duration at about time $t_{m}$ of the measurement, regardless of the value of $x$. It follows that any transition-free period at the point defined by $x$ is essentially a period between times at which measurements are performed, either at that point or anywhere else. During this period, there is no new information about which one of the $\underline{Q}_{k}(\tau, t)$ 's was initially the real quantum matrix $\underline{Q}(x, t)$. The probabilities $w_{k}$ used in Eq. (3.2) do not change. The evolution of the matrix $D(x, t)$ of Eq. (3.3) is due to the evolution of the $\underline{Q}_{k}(x, t)$ 's only. These $\underline{Q}_{t}(x, t)$ 's obey Eq. (3.8). Therefore, between measurements,

$$
\frac{\partial \underline{D}(x, t)}{\partial t}=-i[H \underline{D}(x, t)-\underline{D}(x, t) H]
$$

just as in quantum theory and as expressed by Eq. (2.25) in the translationinvariant formalism. 


\subsection{Collapses of the Quantum Matrix}

Now consider the short transition period of duration $\tau_{m}$ in which $\underline{C}(\tau, t)$ is not zero. During this period $C(x, t)$ is equal to $\lambda_{m}$ times $\pi(x, t)$ given by Eq. (3.7). Equation (3.4) can be integrated. One can express $Q(x, t)$ at any time $t$ in this period knowing its value at time $t_{i}$ at the beginning of the period

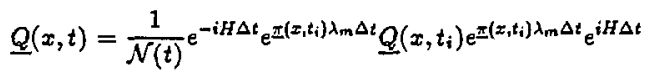

where

$$
\begin{aligned}
t_{i} & =T(x)+t_{m} \\
\Delta t & =t-t_{i}
\end{aligned}
$$

and $\mathcal{N}(t)$ is a normalization factor that keeps

$$
\operatorname{Tr} \underline{Q}(x, t) \equiv 1
$$

Now, at the end $t$, of the transition period,

$$
t_{f}=\tau_{m}+t_{i}=\tau_{m}+T(x)+t_{m}
$$

Between times $t_{m}$ and $t_{i}, \boldsymbol{C}(x, t)$ has been zero, operator $\underline{\pi}(x, t)$ has evolved according to $E q$. (3.7), and the matrix $Q(x, t)$ has evolved according to Eq. (3.8). After $t_{i}, \underline{Q}(x, t)$ has evolved, in the transition period, according to Eq. (3.10). At the end $t_{f}$ of the transition period

$$
Q\left(x, t_{f}\right)=\frac{1}{\mathcal{N}\left(t_{f}\right)} e^{-i H\left(T(x)+r_{m}\right)} e^{\underline{\pi}_{\mu}(x) \lambda_{m} \tau_{m}} Q\left(x, t_{m}\right) e^{\underline{x}_{\mu}(x) \lambda_{m} \tau_{m}} e^{i H\left(T(x)+\tau_{m}\right)}
$$

In the model, we assume that the parameter $\tau_{m}$ is very small, but we also assume the product $\lambda_{m} \tau_{m}$ to be very large. We assume $\tau_{m}$ and $\lambda_{m} T_{m}$ so close to the limits zero and $\infty$, respectively, that the differences between their values and the limits are experimentally unnoticeable. Since $\pi_{\mu}(x)$ is a projection operator with eigenvalues 0 or 1 only,

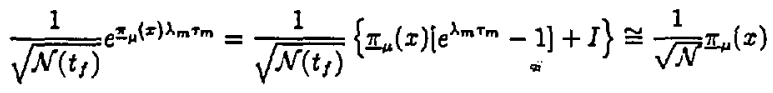


where $\mathcal{N}$ is a normalization factor different from $\mathcal{N}\left(t_{f}\right)$. Equation (3.16) holds except, of course, in rare pathological cases where $\operatorname{Tr}\left[\underline{\pi}_{\mu}(x) \underline{Q}\left(x, t_{m}\right)\right]$ is zero. Except for these pathological cases, Eq. (3.15) can be rewritten for $\lambda_{m} \tau_{m}=\infty$. Assuming values of $\tau_{m}$ equal to an infinitesimal amount $\epsilon$

$$
\underline{Q}\left(x, t_{f}\right)=\underline{Q}\left(x, t_{m}+T(x)+\epsilon\right) \cong \frac{1}{\mathcal{N}} e^{-i H T(x)} \underline{\pi}_{\mu}(x) \underline{Q}\left(x, t_{m}\right) \underline{\pi}_{\mu}(x) e^{i H T(x)}
$$

where the factor $\mathcal{N}$ ensures that the trace condition (3.13) is still valid. Equation (3.17) expresses a collapse of the quantum matrix $Q(x, t)$. If there are points of coordinates $x$ where the pathological case $\operatorname{Tr}\left[\underline{\pi}_{\mu}(x) Q\left(x, t_{\mathrm{m}}\right)\right]=0$ occurs, no divergence is encountered, however, because Eq. (3.15) reduces to

$$
Q\left(x, t_{j}\right)=e^{-i H\left(t_{j}-t_{m}\right)} Q\left(x, t_{m}\right) e^{i H\left(t_{j}-t_{m}\right)}
$$

as if no measurement had been performed.

To make the predictions of the model practically equivalent to those of quantum theory, we need to consider very high values for $V$ such that the discrepancies with the limit $V=\infty$ are not experimentally detecteble. Then $T(x)$ of Eq. (3.5) is zero. Equation (3.17) can be written

$$
Q\left(x, t_{\mathrm{m}}+\epsilon\right) \cong \frac{1}{\mathcal{N}} \pi_{\mu}(x) \underline{Q}\left(x, t_{\mathrm{m}}-\epsilon\right) \underline{\pi}_{\mu}(x)
$$

\subsection{Role of the Spatial-Compatibility Condition in Collapses}

To ensure compatibility between measurements taken at different points $m_{1}$ and $m_{2}$ of coordinates $x_{m 1}$ and $x_{m 2}$ in space, we have to assume that $\underline{Q}(x, t)$ satisfies an initial spatial-cor patibility condition analogous to Eq. (2.26) but which need only be made approximate. Whatever the points $X_{1}$ of coordinate $x_{1}$ and $X_{2}$ of coordinate $x_{2}$ are,

$$
\underline{Q}\left(x_{2}, t\right) \simeq e^{i P\left(x_{2}-x_{1}\right)} \underline{Q}\left(x_{1}, t\right) e^{-i P\left(x_{2}-x_{1}\right)}
$$

When $H$ and $P$ commute as assumed in Ey. (2.23), and if $\mathrm{V}=\infty$, it is not too difficult to show that, if condition (3.20) holds at some initial time $t=t_{0}$, condition (3.20) wili still hold for any time $t>t_{0}$ whether or not 
collapses occur in the interval $t_{0} t$. For finite values of the constants $V, \lambda_{m}$, and $\tau_{m}$, the condition will also hold later except at times $t>t_{0}$, where a transition generated by some measurement, somewhere, has induced a collapse at point $X_{1}$ but not yet at point $X_{2}$, or vice versa. This conservation property is demonstrated for any value of $V$ in Appendix $B$. Therefore, condition (3.20) has only to be imposed as an initial condition before any measurement is made in order later to be valid almost always. It ensures that in the model with $V=\infty$ the density matrix $D(x, t)$ of Eq. (3.3) also satisfies the same compatibility condition is $\mathrm{Eq}$. (2.26) in quantum theory.

Equation (3.20) allows us to write an expression for the normalization constant $\mathcal{N}$ in Eq. (3.19) at points located at some distance $x-x_{m}$ from the point of measurement $m$. Using Eqs. (2.21) and (3.1)

$$
\mathcal{N}=\operatorname{Tr}\left[\underline{\pi}_{\mu}(x) \underline{Q}\left(x, t_{m}-\epsilon\right)\right] \simeq \operatorname{Tr}\left[\Pi_{\mu} \underline{Q}\left(x_{m}, t_{m}\right)\right]=\wp_{\mu}
$$

The uncertainties we have about $\underline{Q}(x, t)$ have to be folded in. After a measurement made by an observer there is new information about which one of the $\underline{Q}_{k}(x, t)$ was, before measurement, the real quantum matrix $\underline{Q}(x, t)$. The probabilities $w_{k}$ that the renl quantum state matrix $\underline{Q}$ was actually equal to the matrix $\underline{Q}_{k}$ are changed soon after the time $t_{m}$ of the measurement. The time delay between measurement and transmission of information to the observer is unimportant in the model. For ine saive of simplicity, the mathematical formulae expressing the evolution of the $w_{k}$ 's are written as if the $w_{k}$ 's changed at the time of the collapse. Thus there is only one discontinuity in the evolution of $D(x, t)$, at the same time as $\underline{Q}(\tau, t)$ collapses.

Given the conditional probabilities $\varphi_{k_{\mu}}$ that the apparatus measures the result $\psi$ when the quantum matrix is $\underline{Q}_{k \mu}$, and using Eq. (3.2),

$$
w_{k}(t+\epsilon)=\frac{w_{k}(t-\epsilon) \wp_{k \mu}}{\sum w_{k^{\prime}}(t-\epsilon) \wp_{k^{\prime} \mu}}=\frac{w_{k}(t-\epsilon) \wp_{k \mu}}{\mathcal{P}_{\mu}}
$$

The evolution of the density matrix $D(x, t)$ over the t:ansition period can be obtained using Eqs. (3.3), (3.19), (3.22), and (3.21) 


$$
\begin{aligned}
\underline{D}(x, t+\epsilon) & =\sum_{k} w_{k}(t+\epsilon) \underline{Q}_{k}(x, t+\epsilon) \\
& =\sum_{k} \frac{w_{k}(t-\epsilon) \varphi_{k \mu}}{\mathcal{P}_{\mu}} \frac{\underline{\pi}_{\mu}(x) \underline{Q}_{k}\left(x, t_{m}-\epsilon\right) \underline{\pi}_{\mu}(x)}{\varphi_{k \mu}} \\
& =\frac{1}{\mathcal{P}_{\mu}} \underline{\pi}_{\mu}(x) \underline{D}\left(x, t_{m}-\epsilon\right) \underline{\pi}_{\mu}(x)
\end{aligned}
$$

Equation (3.23) is equivalent to Eq. (2.20), and therefore the collapse of $D(x, t)$ is the same in the model as in quantum theory.

To justify the spatial-compatibility condition one could assume that, at the beginning of the universe, the quantum matrix $Q(x, t)$ was independent of $x$ and commuted with $P$ to an acceptable accuracy. The spatialcompatibility condition was holding then. Later, many events analogous to measurements, i.e., inducing collapses as described by Eq. (3.15), occurred spontaneously. The commutation relation between $P$ and $Q$ was destroyed whenever $\underline{\Pi}_{\mu}$ did not commute with $P$, but the spatial-compatibility condition was conserved, as shown in Appendix B. Another possible justification relies on the property, shown in Appendix C, that cọndition (3.20) is reached automatically after a larger number of collapses ruled by Eq. (3.15) under a wide range of initial circumstances. In either case, what has to be assumed is that many phenomena generating collapses have taken place in the remote past. These collapse phenomena would have acted like "measurements" but would not necessarily need to be actual measurements performed by human beings. For instance, these phenomena could have been spontaneous measurements of the type considered in Section 5.3.

There may be other scenarios by which that condition could have been generated in the universe.

\subsection{Collapses Without Observers}

Regardless of a possible justification of the spatial-compatibility condition, the model allows for the existence of events having the same effect as "measurements" but at a time when there were no observers to perform actual measurements. Though the model does not require that such events 
exist or have existed, we now consider the possibility that guch events are still occurring and anaiyze the consequences of such an assumption.

There are collapses according to Eq. (3.19) with probabilities given by Eq. (3.1) but we do not know the outcome $\mu$. To the uncertainties $w_{k}$ we had about the $\underline{Q}_{k}(x, t)$ 's before measurement, we have to add the uncertainties $\varphi_{k \mu}$ as to which outcome $\mu$ resulted from the collapse phenomenon. The combined probability is

$$
w_{k \mu}(t+\epsilon)=w_{k}(t-\epsilon) p_{k \mu}
$$

The density matrix after the collapse phenomenon can be computed using Eqs. (3.3), (3.19), (3.21), (3.24)

$$
\begin{aligned}
D(x, t+\epsilon) & \simeq \sum_{k_{1, \mu}} w_{k \mu}(t+\epsilon) \frac{\underline{\pi}_{\mu}(x) \underline{Q}_{k}\left(x, t_{m}-\epsilon\right) \underline{\pi}_{\mu}(x)}{\varphi_{k_{\mu}}} \\
& =\sum_{\mu} \underline{\pi}_{\mu}(x) \underline{D}\left(x, t_{m}-\epsilon\right) \underline{\pi}_{\mu}(x) \\
& =\sum_{\mu} \mathcal{P}_{\mu} \frac{\underline{\pi}_{\mu}(x) \underline{D}(x, t-\epsilon) \underline{\pi}_{\mu}(x)}{\operatorname{Tr}\left[\underline{\pi}_{\mu}(x) D(x, t-\epsilon) \underline{\underline{m}}_{\mu}(x)\right]}
\end{aligned}
$$

just as if, in quantum theory, a measurement is performed at time $t_{m}$ and we are not told what the result $\mu$ is.

Equation (3.25) implies a fundamentally different evolution for $D(x, t)$ than Eq. (3.9), which describes evolution in the absence of measurements. The solution to Eq. (3.9) is a unitarity transformation for $\underline{D}(x, t)$. The solution of Eq. (3.25) is not. Therefore, if there were spontaneous "collapse "phenomena" acting like measurements, it would change the predictions for some isolated systems in a way that may happen to be detectable experimentally. ${ }^{[9]}$ Since the model is compatible with such phenomena, the limit to their frequency can only come from past experiments. ${ }^{110]}$ In Section 5.3 we consider a possible use of such phenomena in measurement theory. 


\section{PRoperties RELATED TO LOCALITY}

So far, we have considered extreme values for perameters $V, \lambda_{m}$, and $\tau_{m}$, which are involved in Eq. (3.6), in order to show that the model can generate the same predictions as quantum theory. The propagation velocity $V$ of the transition has to be much higher than the velocity of light $c$; the duration $\tau_{m}$ of the transition period has to be small, but the dimensionless product $\lambda_{m} \tau_{m}$ of parameters $\lambda_{m}$ and $\tau_{m}$ has to be much larger than 1 . For extreme values of $V, \tau_{\mathrm{m}}$, and $\lambda_{\mathrm{m}} \tau_{\mathrm{m}}$, equivalence of the predictions was shown in Section 3. It results from the equivalence of Eqs. (3.2), (3.9), and (3.23) for the model and Eqs. (2.18), (2.25), and (2.20), respectively, for quantum theory. In addition, we need the spatial-compatibility condition (3.20) as an initial condition to generate the same resulis as the initial condition (2.26) in quantum theory.

In this section we investigate the properties of the model when parameter $V$ is not infinity. It gives the model its locality property and allows for some possible discrepancies with the predictions of quantum theory. If $V$ is not too high these discrepancies will be experimentally detectable.

\subsection{Invariances and Locality of the Model}

As stated at the beginning of Section 3, the description of reality ir: the model relies only on the following quantities:

a) the observables $\mathcal{M}$ and operators $M$, which we need to define projection operators $\Pi_{\mu}$ that determine the probabilities $p_{\mu}$ of Eq. (3.1) at the time of the measurements;

b) operators $Q(x, t)$ and $C(x, t)$ defined as functions of space coordinates $x$ and time $t$

c) operators $P$ and $H$, which are constants independent of location and time. They are available everywhere.

An observable $\mathcal{M}$ is a quantity that manifests itself in the measuring apparatus at point $m$. It is a quantity that it is natural to attach to the location $m$ of the apparatus, regardless of the origin of the space coordinates. When defined as such, it is a translation-invariant and local quantity. Op- 
erator $\underline{M}$ for the model is the same as operator $\underline{M}$ defined by Eq. (2.16) for quantum theory. it is an operator that is independent of the location of the origin of the space coordinates and does not change if the apparatus is moved. It is a translation-invariant quantity describing the characteristics of the apparatus at point $m$ of cocrdinates $x_{m}$ at time $t_{m}$. Operator $M$, and thus the $\Pi_{\mu}$ 's, are characteristic quantities of objects located at point $m$. Operators $\underline{Q}(x, t)$ and $\underline{C}(x, t)$ are defined as quantities describing reality at the point having coordinates $x$ at time $t$. They are also defined as translation invariant. The model is translation invariant and local if there is nc contradiction between these definitions and all the equations it uses.

Like $M$ and the $\Pi_{\mu}$ 's, $Q\left(x_{m}, t_{m}\right)$ is a quantity defined at point $m$. The probability $\wp_{\mu}$ of Eq. (3.1) to measure $\mathcal{M}=\mu$ at point $m$ depends only on translation-invariant quantities defined at $\mathrm{m}$. Equation (3.1) is consistent with the translation invariance and locality of the model.

Since $C(x, t)$ is a translation-invariant quantity defined at $x$ and $t$, and $H$ is a constant available over the entire space-time, the evolution of $Q(x, t)$ via Eq. (3.4) depends only on translation-invariant quantities available at $x$ and $t$. This evolution (3.4) is translation invariant and local. The evolution of $C(x, t)$ of Eq. (3.6) can be expressed by a partial derivative equation, as shown in Appendix A. In that equation, (A.17), the evolution of $C(x, t)$ is almost everywhere determined by products of $B$ and $P$ with $C(x, t)$ for the same $x$ and $t$ and by its spatial derivatives, which depend on the vilues of $C(x, t)$ in the neighborhood. Only at the point of measurement $m$ does it also depend on $\Pi_{\mu}$, but $\Pi_{\mu}$ is a translation-invariant operator associated to puint $m$. Therefore, the evolution of $\underline{C}(x, t)$ is also translation invariant and local.

As mentioned in Section 3.4, the initial spatial-compatibility condition (3.20) can be generated in scenarios involving a large number of events analogous to meesurements. These events would be local phenomena in the past like measurements today.

For $V \neq c_{1}$ the model cannot be Lorentz invariant because then expression (3.6) of $C(x, t)$ is not Lorentz invariant. The functions $\Theta$ in Eq. (3.6) define a region of space-time where $C(x, t)$ is nonzero using the values $t, t_{m}$, $x$, and $x_{m}$ deined in the privileged restframe only. Equation (3.6) would define a different nonze:o region for $C(x, t)$ if it were applied in a lifferent Lorentz restfras te. However, in the privileged restframe, the model is 
rotation invariant.

Let $R(\omega)$ be the operator that expresses the transformation of an element $\Psi$ of Fock space when the space coordinates are rotated by an angle $|\omega|$ around the axis defined by the vector $\omega ; \Psi \rightarrow R(\omega) \Psi$. The operator $R(\omega)$ is unitary. The quantities that describe measurements and evolutions transform like operators in Fock Space: $M \rightarrow R M R^{-1}, \Pi_{\mu} \rightarrow R \prod_{\mu} R^{-1}$, $P \rightarrow R P R^{-1}$, and $H \rightarrow R H R^{-1}=H$ since these operators are the same in the model as in quantum theory. We will give $\underline{Q}$ and $C$ the properties $Q \rightarrow R Q R^{-1}$ and $C \rightarrow R C R^{-1}$. It follows that Eqs. (3.1) and (3.4) can be written for the transformed quantitieg as well as for the original ones. Equation (3.3) gives a density matrix $D$ that can be equated to the one of quantum theory in either coordinate system. The quantity $T(x)$ of Eq. (3.5) is rotation invariant in the privileged restrame. Therefore, Eq. (3.6) can be written using the rotated quantities as well. The spatial-compatibility condition (3.20) for all $x_{1}$ 's and $x_{2}$ 's implies the same relationship between the transformed quantities and the original ones. It follows that the model is rotation invariant in the privileged restframe.

The model has the same properties as a classical tensorial field theory in three dimensions. It is translation invariant because it pictures reality using only quantities that do not depend on the choice of origin of the spatial coordinates. It is rotation invariant because, in rotations, these quantities are transformed according to formulae that leave the equations identical to what they were before. It is local because these quantities are attached to points in space, and their equations of evolution can be written as bounds between quantities defined at the same point and in the neighborhood only.

\subsection{Evolution When $V$ Is Not Infinite}

In the following sections we want to analyze some of the properties of the predictions of the model if the value of the parameter $V$ is not so extremely high as assumed in Section 3. We may now expect deviations for quantum-theory predictions. The duration $\tau_{m}$ of the transition period is still going to be vanishingly small. The product $\lambda_{m} \tau_{m}$ will still be extremely large. We will take the limit

$$
\lambda_{m} \tau_{m}=\infty
$$


In the transition-free period the evolution of $\underline{Q}(x, t)$ is ruled by Eq. (3.8). Consider two times to and $t$ preceding time $t_{i}$ at which the transition zone generated by the measurement at time $t_{m}$ reaches the point defined by the coordinate $x$,

using Eqs. (3.11) and (3.5). Then

$$
t_{0}<t<t_{i}=t_{m}+T(x)=t_{m}+\frac{\left|x-x_{m}\right|}{V}
$$

$$
\underline{Q}(x, t)=e^{-i H\left(t-t_{0}\right)} \underline{Q}\left(x, t_{0}\right) e^{i H\left(t-t_{0}\right)}
$$

The collapse of the quantum matrix $\underline{Q}(x, t)$ over the transition period ending at time/ $t$, is expressed by Eq. (3.17), which was written before we used the limit $/ V=\infty$ in the collapse equations.

$$
\underline{Q}\left(x, t_{\rho}\right) \cong \frac{1}{\mathcal{N}} e^{-i H\left(t_{f}-t_{m}\right)} \underline{x}_{\mu}(x) \underline{Q}\left(x, t_{m}\right) \underline{\pi}_{\mu}(x) e^{i H\left(t_{j}-t_{m}\right)}
$$

where the/ normalization factor $\mathcal{N}$ keeps the trace of $\underline{Q}$ equal to one.

$$
\mathcal{N}=\operatorname{Tr}\left[\underline{\pi}_{\mu}(x) \underline{Q}\left(x, t_{m}\right)\right]
$$

Before and after the transition period, the quantum matrix evolves according to Eq. (3.8). Consider a time $t_{0}$ before and another time $t$ after the transition period.

$$
\begin{gathered}
t>t_{f}=t_{i}+\epsilon \cong t_{m}+T(x)=t_{m}+\frac{\left|x-x_{m}\right|}{V}>t_{0} \\
\underline{Q}(x, t)=\frac{1}{\mathcal{N}} e^{-i H\left(t-t_{m}\right)} \underline{\pi}_{\mu}(x) e^{-i H\left(t_{m}-t_{0}\right)} \underline{Q}\left(x, t_{0}\right) e^{i H\left(t_{m}-t_{0}\right)} \underline{\pi}_{\mu}(x) e^{i H\left(t-t_{m}\right)}
\end{gathered}
$$

Expression (4.7) of $\underline{Q}(x, t)$ after the transition period does not depend on $V$ or any function of $\vec{V}$. Therefore, $\underline{Q}(x, t)$ is independent of the value of the parameter $V$ as long as $V$ is in the range where the inequality $t>t_{f}$ in $(4.6)$ is satisfied. If $V$ does not satisfy this inequality, and $\tau_{m}$ is negligible, then $\underline{Q}(x, t)$ is given by Eq. (4.3). 


\subsection{Measurements Well Spaced in Time}

Consider two measurements performed at points $m_{1}$ and $m_{2}$ having coordinates $x_{m 1}$ and $x_{m 2}$ at times $t_{m 1}$ and $t_{m 2}>t_{m 1}$. We define

$$
\begin{aligned}
\Delta t & =t_{m 2}-t_{m 1} \text { by definition assumed to be }>0 \\
\Delta x & =x_{m 2}-x_{m 1}
\end{aligned}
$$

Let us assume that these two measurements are well spaced in time so that the transition zone can propagate from $m_{1}$ to $m_{2}$ in the time interval $\Delta t$

$$
\Delta t>\frac{|\Delta x|}{V}
$$

These conditions apply to the case of Fig. 1 where the second measurement occurs in the light cone of the first. They also apply to the case of Fig. 2, despite the fact that the two measurements are outside the light cones of one another.

The quantum matrix $Q\left(x_{m 2}, t_{m 2}\right)$ is given by Eq. (4.7). It is the same as if $V$ were extremely large. The probabilities $\wp_{\mu 2}$ calculated by Eq.(3.1) will also be exactly the same as if $V$ were extremely large.

After time $t_{m 2}$, a second transition zone propagates from point $m_{2}$ at coordinates $x_{m 2}$. Consider any point $X$ of coordinates 2 ; it sees two transition periods, one at $t_{x 1}$ due to the measurement at $m_{1}$ and another at tine $t_{x 2}$ due to the measurement at $m_{2}$

$$
\begin{aligned}
& t_{x 2}=t_{m 2}+\frac{\left|x-x_{m 2}\right|}{V} \\
& t_{x 1}=t_{m 1}+\frac{\left|x-x_{m 1}\right|}{V}
\end{aligned}
$$

Using Eģs. (4.8), (4.9), and (4.10) and a triangle inequality on the triangle formed by the points of coordinates $x, x_{m 1}$, and $x_{m 2}$, we obtain

$$
\begin{aligned}
t_{x 2} & =t_{m 2}-t_{m 1}+t_{x 1}-\frac{\left|x-x_{m 1}\right|}{V}+\frac{\left|x-x_{m 2}\right|}{V} \\
& >t_{x 1}+\frac{|\Delta x|}{V}+\frac{\left|x-x_{m 2}\right|}{V}-\frac{\left|x-x_{m 1}\right|}{V} \\
& >t_{x 1}
\end{aligned}
$$


It follows that the transition period due to the second measurement occurs at the point of coordinates $x$ at a time $t_{x 2}$ after the transition period due to the first measurement has ended. Any tirse in between, the matrix $Q(x, t)$ has seen only the collapse due to the first measurement. It is expressed by Eq. (4.7). It is the same as if $V$ were infinite. The same argument applies for the collapse occurring at $t_{x 2}$ due to the second measurement. Any time after $t_{x 2}$, the matrix $\underline{Q}(x, t)$ is the same as if parameter $V$ were infinite.

If the measurement processes are well spaced in time, i.e., if the time $\Delta t$ elapsed between them and the distance $|\Delta x|$ between their locations satisfy inequality (4.10), the probabilities $p_{\mu 2}$ of measurement results at $m_{z}$ and the quantum matrices $Q(x, t)$ anywhere, any time, after the second transition period has ended, are the same as if parameter $V$ were infinite. In Section 3 it has been shown that the predictions of the model for extremely large values of $V$ are the same as the ones of quantum theory. It follows that the predictions of the model for any finite values of parameter $V$ are exactly the same as the ones of quantum theory, with only the additional condition that the measurements are so well spaced in time that the time $\Delta t$ and the distance $\Delta x$ between them satisfy inequality (4.10). No contradictions between the model and quantum theory could have been detected in any of the experiments where successive measurements are separated by time intervals satisfying that inequality.

\subsection{Measurements Closely Spaced in Time}

We now consider two measurements so closely spaced in time that the transition does not have time to propagate from the first point of measurement $m_{1}$ at $t_{m 1}$ to the second one $m_{2}$ at $t_{m 2}$ at a distance $\Delta x$ in the time $\Delta t$ that separates them (this is the case sketched in Fig. 3). Using the definitions of Eq. (4.8) and (4.9)

$$
\Delta t<\frac{|\Delta x|}{V}<\frac{|\Delta x|}{c}
$$

During the period $\Delta t$, the matrix $Q\left(x_{m 2}, t\right)$ obeys Eq. (4.3). Therefore, the probability $\emptyset_{\mu_{2}}$ of an outcome $\mu_{2}$ at $m_{2}$ is a function $r_{2}\left(\beta_{2}, \mu_{2}\right)$ of the characteristics $\beta_{2}$ of the apparatus at $m_{2}$ and of the variable $\mu_{2}$, independent 
of the characteristics $\beta_{1}$ of the apparatus at $m_{1}$ and of the value of the outcome $\mu_{1}$ of the measurement at $m_{1}$.

$$
p_{\mu 2}=r_{2}\left(\beta_{2}, \mu_{2}\right)=\operatorname{Tr}\left[\underline{\Pi}_{\mu_{2}}\left(\beta_{2}\right) e^{-i H \Delta t} \underline{Q}\left(x_{m 2}, t_{m 1}\right) e^{i H \Delta t}\right]
$$

This expression of the probability $p_{\mu 2}$ of the outcome $\mu_{2}$ is the same as if the first measurement had not been performed.

The probability $\wp_{\mu 1}$ of the outcome $\mu_{1}$ at $m_{1}$ is a function $r_{1}\left(\beta_{1}, \mu_{1}\right)$ of $\beta_{1}$ and of $\mu_{1}$, which can be derived from Eq. (3.1), of course independent of the characteristics $\beta_{2}$ and of the outcome $\mu_{2}$ of the measurement at $m_{2}$, which will be performed later. The joint probability for the outcomes $\mu_{1}$ at $m_{1}$ and $\mu_{2}$ at $m_{2}$ is a function

$$
\wp_{\mu_{1} \mu_{2}}=r\left(\beta_{1}, \beta_{2}, \mu_{1}, \mu_{2}\right)=r_{1}\left(\beta_{1}, \mu_{1}\right) \quad r_{2}\left(\beta_{2}, \mu_{2}\right)
$$

where

$$
\wp_{\mu_{1}}=r_{1}\left(\beta_{1}, \mu_{1}\right)=\operatorname{Tr}\left[\underline{\Pi}_{\mu_{1}}\left(\beta_{1}\right) \underline{Q}\left(x_{m 1}, t_{m 1}\right)\right]
$$

To get predictions from the model, the observer's uncertainties as to what the real matrix $Q(x, t)$ is originally have to be folded in. Let us use the definitions of $w_{k}$ and $\underline{Q}_{k}(x, t)$ given in Section 3.1. There are functions $r_{1 k}\left(\beta_{1}, \mu_{1}\right)$ and $r_{2 k}\left(\beta_{2}, \mu_{2}\right)$ of $\mu_{1}$ and $\mu_{2}$ defined by using $Q_{k}\left(x_{m 1}, t_{m 1}\right)$ and $\underline{Q}_{k}\left(x_{m 2}, t_{m 1}\right)$ instead of $\underline{Q}\left(x_{m 1}, t_{m 1}\right)$ and $\underline{Q}\left(x_{m 2}, t_{m 1}\right)$ in Eqs. (4.17) and (4.15), respectively. After the uncertainties $w_{k}$ have been taken into account, the joint probability distribution is

$$
\mathcal{P}_{\mu_{1}, \mu_{3}}=R\left(\beta_{1}, \beta_{2}, \mu_{1}, \mu_{2}\right)=\sum_{k} w_{k} r_{1 k}\left(\beta_{1}, \mu_{1}\right) r_{2 k}\left(\beta_{2}, \mu_{2}\right)
$$

If $\mu_{1}$ and $\mu_{2}$ are dichotomic values, the joint probability function of Eq. (4.18) is of a mathematical form for which it has been demonstrated ${ }^{[5]}$ that Bell's inequalities $[11]$ must be valid. It follows that for measurements satisfying inequality (4.14), the joint probability distributions for different settings $\beta_{1}$ and $\beta_{2}$ of the apparatus must satisfy Bell's inequalities.

There are cases of measurements located and timed as in Fig. 3 for which the predictions of quantum theory violate Bell's inequalities. Therefore, when $V$ is not too large and measurements satisfying inequality (4.14) are possible, there are cases where the predictions of the model must differ from the predjctions of quantum theory. Such circumstances provide a 
ground for relevant tests of quantum theory. They involve distant apparati and short time delays between measurements. The ratio between the spatial separations $\Delta x$ and the time delays $\Delta t$ has to be not only larger than the velocity of light $\varepsilon$ but also larger than velocity $V$. Of course, $\Delta t$ and $\Delta x$ are the values of the time and the space interval between times and points of measurement in this privileged space-time restframe, for which the evolution equations (3.4) and (3.6) are valid.

The predicted violation of quantum theory is not caused by some specific detail of the model developed here. Under a very broad set of hypotheses, it has been demonstrated that the probability distributions of the observables at two points $m_{1}$ and $m_{2}$ must satisfy Bell's inequalities if no influence can be exerted between $m_{1}$ and $m_{2},{ }^{[4],[3]}$ In any model where there is an upper bound $V$ to the velocity of propagation of all physical effects, it is possible to define points $m_{1}$ and $m_{2}$ in space-time such as the ones in Fig. 3 between which no influence can be exerted. In any such model, there are violations of quantum theory. It seems likely to me that such an upper bound $V$ indeed exists in nature. Therefore, it is my speculation that such violations of quantum theoretical predictions do exist. They involve almost simultaneous measurements at distance in a privileged space-time restframe.

The evolution of the state matrices $Q$ after two meavurements closely spaced in time is described in Appendix $\bar{D}$.

\subsection{Isolated Systems}

To make more accurate calculations of the predictions of the model, we will consider, as we did in Section 2.1, the case of isolated quantum systems $S$ that are described by fewer variables than all the particles of the universe. For this purpose, we do not even have to include the apparatus variables in the system $S$. For each value of the index $k$ of the matrix $Q_{k}(x, t)$, we define the operator

$$
Q_{u k}\left(t_{m 1}\right)=e^{-i P_{m 2}} \underline{Q}_{t}\left(x_{m 1}, t_{m 1}\right) e^{i P_{m+1}}
$$

Because of the spatial-compatibility condition (3.20) before the first 
measurement

$$
Q_{u k}\left(t_{m 1}\right) \cong e^{-i P_{m} 2} \underline{Q}_{k}\left(x_{m 2}, t_{m 1}\right) e^{\mathrm{i} P_{x_{m} 2}}
$$

Then, from $Q_{u k}$ we define another operator $Q_{S k}$ in the space of the variables $S$ of the system, using a trace operation as in Eq. (2.2)

$$
Q_{S k}\left(t_{m 1}\right)=\operatorname{Tr}_{\operatorname{ext}(S)} Q_{u k}\left(t_{m 1}\right)
$$

Operators $\Pi_{S, \mu}, \Pi_{\mu}$, and $\Pi_{\mu}$ have been defined so that $\mathcal{P}_{\mu}$ from Eq. (2.18) using $D$ and $\mathcal{P}_{\mu}$ from Eq. (2.4) using $D_{S}$ are the same. The only condition is that the matrices $D_{S}, D_{u}$, and $\underline{D}$ satisfy Eqs. (2.2) and (2.19). Equations (4.19), (4.20), and (4.21) express the same relationship between $\underline{Q}_{k}$ and $Q_{S k}$ as Eqs. (2.19) and (2.2) between $D$ and $D_{s}$. First, Eqs. (4.17) and (4.15) can be written for $r_{1 k}\left(\beta_{1}, \mu_{1}\right)$ and for $r_{2 k}\left(\beta_{2}, \mu_{2}\right)$ adding an index $k$ to the symbols $\varphi, r$, and $Q$. Then they can be shown to be equivalent to the following expressions:

$$
\begin{aligned}
& r_{1 k}\left(\beta_{1}, \mu_{1}\right)=\operatorname{Tr}_{S}\left[\Pi_{S_{\mu_{1}}}\left(\beta_{1}\right) Q_{S k}\left(t_{m 1}\right)\right] \\
& r_{2 k}\left(\beta_{2}, \mu_{2}\right)=\operatorname{Tr}_{S}\left[\Pi_{S_{\mu_{2}}}\left(\beta_{2}\right) e^{-i H_{S} \Delta t} Q_{S k}\left(t_{m 1}\right) e^{i H_{S} \Delta t}\right]
\end{aligned}
$$

where $H_{S}$ is the Hamiltonian in the system variables $S_{i} H_{S}$ determines the evolution of $D_{S}$ between measurements. Such an operator $H_{S}$ can be defined when the system $S$ is isolated, i.e. for states of the universe such that the effect of the hamiltonian $H$ can be approximated as

$$
H \cong H_{S} \times I_{e x t(S)}+I_{S} \times H_{e x t(S)}
$$

Since the translation-invariant matrices $\underline{D}(x, t)$ of quantum theory, defined by Eq. (2.19), have been equated to the matrices $D(x, t)$ defined by Eq. (3.3) for the model, we can also equate the density matrix $D_{S}\left(t_{m 1}\right)$ of quantum theory in the system's variables, defined by Eq. (2.2), to the matrix $D_{S}\left(t_{m 1}\right)$ defined for the model

$$
D_{S}\left(t_{m 1}\right)=\sum_{k} w_{k} Q_{S k}\left(t_{m 1}\right)
$$

This Inatrix permits us to compute the probability $R_{1}\left(\beta_{1}, \mu_{1}\right)$ of the outcome $\mu_{1}$ of the first measurement at $t_{m 1}$. It also allows us to compute 
the probability $R_{2}\left(\beta_{2}, \mu_{2}\right)$ of the outcome $\mu_{2}$ in the second measurement under the assumption that the first one has not been performed. Using Eqs. (2.4), (4.25), (4.23), and (4.22)

$$
\begin{aligned}
R_{1}\left(\beta_{1}, \mu_{1}\right) & =\operatorname{Tr}_{S}\left[\Pi_{S \mu_{1}}\left(\beta_{1}\right) D_{S}\left(t_{m 1}\right)\right]=\sum_{k} w_{k} r_{1 k}\left(\beta_{1}, \mu_{1}\right) \\
R_{2}\left(\beta_{2}, \mu_{2}\right) & =\operatorname{Tr}_{S}\left[\Pi_{S \mu_{1}}\left(\beta_{2}\right) e^{-i H_{4} \Delta t} D_{S}\left(t_{m 1}\right) e^{i H_{S} \Delta t}\right] \\
& =\sum_{k} w_{k} r_{2 k}\left(\beta_{2}, \mu_{2}\right)
\end{aligned}
$$

If the system under study is in a state called a pure case of quantum theory, i.e., if the matrix $D_{S}$ is of the form of Eq. (2.3), Eq. (4.25) and the positiveness of the $Q_{s k}$ require that

$$
Q_{s k}\left(t_{m 1}\right)=D_{S}\left(t_{m 1}\right) \text { for all } k^{\prime} s
$$

Then, using Eqs. (4.22), (4.23), (4.26), (4.27), and (4.18) the joint probability distribution for $\mu_{1}$ and $\mu_{2}$ is obtained

$$
R\left(\beta_{1}, \beta_{2}, \mu_{1}, \mu_{2}\right)=R_{1}\left(\beta_{1}, \mu_{1}\right) R_{2}\left(\beta_{2}, \mu_{2}\right)
$$

For pure cases and measurements satisfying condition (4.14), the joint probability distribution is an uncorrelated distribution of $\mu_{1}$ and $\mu_{2}$ of the form of Eq. (4.29). The functions $R_{1}\left(\beta_{1}, \mu_{1}\right)$ and $R_{2}\left(\beta_{2}, \mu_{2}\right)$ are equal to the probabilities for the outcome $\mu_{1}$ at $m_{1}$ and $\mu_{2}$ at $m_{2}$, respectively, integrated over the possible results at the other point. They are obtained from $D_{S}\left(t_{m 1}\right)$ using Eqs. (4.26) and (4.27). They are also the integrated probabilities predicted by quantum theory.

The pure cases are not the only cases where correlation-free Eq. (4.29) applies. If all the matrices $Q_{S k}$ are not of rank one but are equal to one another, they are also equal to $D_{S}$. These cases will be referred to as improper mixtures because they include the cases defined as improper mixtures in the literature. ${ }^{\left[{ }^{[2]}\right.}$ All the matrices $Q_{s k}$ are equal to one another, because the ambiguities $w_{k}$ we have about the real quantum matrix $\underline{Q}(x, t)$ do not concern the states of the system $S$ but only the variables ext $(S)$ external to the system. Equations (4.22), (4.23), (4.26), (4.27) and (4.18) give a joint probability $R\left(\beta_{1}, \beta_{2}, \mu_{1}, \mu_{2}\right)$ satisfying Eq. (4.29). The functions $R_{1}\left(\beta_{1}, \mu_{1}\right)$ and 
$R_{2}\left(\beta_{2}, \mu_{2}\right)$ are equal to the predictions for single measurements, Eqs. (4.26) and (4.27), both in the model and in quantum theory. Note that the rarik of $D_{S}$ and the $Q_{S k}$ 's can be higher than one for many reasons. For instance, in the cases considered in Appendix $\mathrm{C}$ where the matrices $Q(x, t)$ would be nearly equal to matrices of rank one, the trace operation of Eq. (4.21) can generate a rank of the matrices $Q_{S k}$ that is higher than one by the phenomenon called improper mixture in the literature. ${ }^{[13]}$

For pure cases and improper mixtures, the determination of the final state after the two measurements is also simplified. Using the results obtained in Appendix $\mathrm{D}$, we find that the system density matrix $D_{S}(t)$ in the final state is the same as the one we would have using quantum theory under the condition that the results are also $\mu_{1}$ and $\mu_{2}$.

In the cases where all the matrices $Q_{S k}$ are not the same, i.e., when our ambiguities $w_{k}$ about the real matrices $Q(x, t)$ would also concern the variables of the system $S$, Eq. (4.29) will not apply in general. These cases are referred to as proper mixtures. ${ }^{[12]}$ Each possibility labeled $k$ has to be considered separately, and the joint probability function $R\left(\beta_{1}, \beta_{2}, \mu_{1}, \mu_{2}\right)$ has to be computed from Eqs. (4.18), (4.22) and (4.23). Some correlations in the joint probability distributions may result from this sort of computation, but, of course, Bell's inequalities ${ }^{[4],[s]}$ still must be satisfied.

\subsection{Superluminous Communication}

In relativistic quantum theory, superluminous communication is not possible ${ }^{[13]}$ because the probability distribution of any set of observables measurable by one observer at a point $m$ in space-time, integrated over all correlations with observables elsewhere, is independent of any parameter that could be set by another observer outside the past light cone of point $m$. Superluminous communication is also impossible in the model in the limit of $V$ being infinite since then the predictions are the same as in quantum theory. It is also impossible if $V$ is finite but with measurements well spaced in time (Fig. 2) since, as we have shown in Section 4.3, the predictions are the same as if $V$ were infinite.

Now let us consider the case of closely spaced measurements sketched in Fig. 3 . The distance $\Delta x$ and the time interval $\Delta t$ between the two 
measurements satisfy inequality (4.14). The probability $\wp_{\mu_{2}}$ of the outcome of $\mu_{2}$ at $m_{2}$ is given by Eq. (4.15). As was stated in Section 4.4, it is the same as if no measurement had been performed at $m_{1}$. By changing the experimental setup at $m_{1}$, no information can be communicated to the observer at $m_{2}$.

In more general terms in the model, no physical effect can propagate at a velocity larger than parameter $V$. It follows that the speed of information transfer between observers is also limited to the absolute maximum $V$. However, there are ways by which a signal could be sent by one observer to another at a speed smaller than $V$ but larger than $c$.

Operations using two measurements will fall into one of the three categories sketched in Figs. 1, 2, or 3, and in all those cases we have seen that there can be no possible superluminous communication. Superluminous communication requires a minimum of three messurements whose times, $t_{m 1}, t_{m 2}$, and $t_{m 3}$, have to be chosen carefully taking their location into account so that, as sketched in Fig. 4.

$$
\begin{aligned}
t_{m 2}-t_{m 1} & <\frac{\left|x_{m 2}-x_{m 1}\right|}{V} \\
\frac{\left|x_{m 3}-x_{m 1}\right|}{V} & <t_{m 3}-t_{m 1}<\frac{\left|x_{m 3}-x_{m 1}\right|}{c} \\
\frac{\left|x_{m 3}-x_{m 2}\right|}{V} & <t_{m 3}-t_{m 2}
\end{aligned}
$$

We will show the possibility of superluminous communication using an example. Consider an initial state composed of three spin-1/2 particles, numbered 1,2 , and 3 , which will be detected at $m_{1}, m_{2}$, and $m_{3}$, where their spins will be measured at times $t_{m 1}, t_{m 2}$, and $t_{m 3}$, as shown in Fig. 4. The spin states are correlated, and we assume that the initial state is a pure state represented by the spin wave function

$$
\Psi\left(t_{0}\right)=\alpha(\Uparrow \uparrow \downarrow+\Downarrow \downarrow \downarrow)+\alpha^{\prime}(\Uparrow \downarrow \uparrow+\downarrow \Uparrow \Uparrow)
$$

where for every particle the arrow $\Uparrow$ means spin-up state and the arrow $\Downarrow$ spin-down state. Three arrows in a row represent the state of the system of the three particles 1,2 , and 3 with their spin values indicated by the three arrows in the same order 1,2, and 3. The terms $\alpha$ and $\alpha$ are nonzero 
coefficients. We assume

$$
|\alpha| \neq\left|\alpha^{\prime}\right|
$$

By applying the rules spelled out in Section 4.5, the probabilities can be computed. For the first measurement the probability is 50/50 to find the first particle with a spin $\uparrow$ or $\downarrow$, as shown by using Eq. (4.26). For the second measurement in the case sketched in Fig. 4, the probability in the model is the same as in quantum theory if the first measurement had not occurred. It is also $50 / 50$ to find particle 2 with a spin $\Uparrow$ or $\Downarrow$, as shown using Eq. (4.27). The probability of each of the four combinations $\pi \Uparrow, \Uparrow \psi_{1} \Downarrow \Uparrow$, and $\Downarrow \psi$ for the spins of particles 1 and 2 is $25 \%$. In each of these four cases, the final state is the same as in quantum theory if the same spin combination has been measured at $m_{1}$ and $m_{2}$. Therefore, for the two cases where the first two particles have parallel spins, the spin of particle 3 is $\Downarrow$. If the first two particles have been found with opposite spin values, the spin of particle 3 is $\pi$. It follows that, under these experimental conditions, the probability distribution of the outcome of the third measirement is $50 \%$ up and 50\% down after integration over the possible results of the first and second measurements.

If either the first, the second, or both measurements has not been performed, measurements are well spuced in time. The predictions of the model are the same as those of quantum theory. The probabilities for the third measurement are $|\alpha|^{2}$ for spin $\Downarrow$ and $\left|\alpha^{\prime}\right|^{2}$ for spin $\Uparrow$. They are not $50 / 50$ because of hypothesis (4.34). They are different than the probabilities obtained when the first and second measurements have been performed.

Assume an observer at $m_{3}$ is set to measure the spin of particle 3 at $t_{m 3}$. Assime that another observer at $m_{1}$ can choose to measure or not to measure the spin of particle 1 at $t_{m 1}$. Assume the measurement of particle 2 at $m_{2}$ at time $t_{m 2}$ is going to be made in any case. By his decision to perform or not to perform the measurement of particle 1 , the observer at $m_{1}$ has the power to influence the probability distribution of the outcome of the meastirement made by the observer at $m_{3}$ even in the conditions of Fig. 4 , where the two measurements would occur outside of the light cones of one another. This example is given here to show one possible mechanism by which, in the model, communication faster than the velocity of light $c$ can be established. 


\section{IMPACT ON MEASUREMENT THEORY}

In our model, an "observable" (i.e., an observable quantity) and all inputs into the computation (3.1) of the probability of the measurement outcome are local quantities attached to a point $m$ of coordinates $x_{m}$. We still have to specify how point $m$ is selected in the measurement process. The location of this point is one of the characteristics of the measurement, which, in our local model of reality, all have to be dependent real and local quantities only. How point $m$ is selected will be the subject of Section 5 .

In this section, parameter $V$ will be assumed to be extremely large as in Section 3. As to the other parameters involved in Eq. (3.6), $\tau_{m}$ will still be vanishingly small, and the product $\lambda_{m} \tau_{m}$ will still be extremely large. The possible discrepancies such as the ones mentioned in Section 4 between the model and quantum theory will be negligible. Only the effect of our local reality requirement on the measurement process will be investigated here.

\subsection{Locality of Measurement Processes}

Since the measuring apparatus has a finite size $L_{m}$, the measurement and corresponding projection operators are functions of field operators $\varphi_{j}(x)$ at coordinates $x \neq x_{m}$, as can be seen from Eqs. $(2.10)$, (2.11), and (2.12). Because of this, the value of the observable $\mathcal{M}$ is correlated to the values of the fields at points other than the point of measurement $m$. Therefore, in quantum theory, the "observable" may not be considered as a local quantity.

Note that the parameter $L_{m}$ does not have to be the size of that which the observer would normally call his measurement apparatus and which we will call system $A$. If that apparatus $A$ is in interaction with other systems $A^{\prime}$, a measurement performed with one of the systems $A^{\prime}$ can have the sarme effect as a measurement with system $A$. ${ }^{[B]}$ The systems $A^{\prime}$ can be of a much smaller geometrical size than apparatus $A$. In some measurement theories, the systems $A^{\prime}$ could just be small elements of system $A$. Then, for this analysis, parameter $L_{m}$ is the much smaller size of a system $A^{\prime}$ instead of the size of the large apparatus $A$. Following this approach, observables could be made local in quantum theory if $L_{m}$ could be made infinitesimally small. 
Unfortunately infinitesimal sizes $L_{m}$ in Fock space lead to mathematical divergences we do not wish to deal with now. Therefore we can use small but finite values of $L_{\mathrm{m}}$.

In the model, the probability distribution (3.1) of that same observable that is nonlocal in quantum theory depends on translation-invariant operators in Fock space and on the quantum state-matrix $Q\left(x_{m}, t_{m}\right)$ at point $m$ only. This is possible because the matrix $Q\left(x_{m}, t_{m}\right)$ contains the same information as the matrix $Q(x, t)$ for any $z$ as long as the spatial-compatibility condition (3.20) holds. Thus the probability distribution of the field $\varphi_{j}(x)$ at $x \neq x_{m}$ can be obtained from $Q\left(x_{m}, t_{m}\right)$ defined at point $m$. This is the mechanism by which the value of an observable defined by Eqs. (2.10) and (2.12) is determined at point $m$ by the probability distribution (3.1) using only quantities ccnsidered to be local in our model of reality. Observables that may not be seen as local in quantum theory can be defined as local quantities in the model. One can minimize problems and confusion resulting from two concepts of locality by considering only small values for the parameter $L_{m}$, on the order, let us say, of 10 or $100 \AA$. Conflicts between the two concepts of locality are thus limited to the atomic scale.

Just as an example of an observable satisfying the above properties, consider the number of baryons $\mathcal{B}(x, t)$ contained in a small volume $\Omega(x)$ of size $L_{m}$ around the point of coordinates $x$. Inside an apparatus, $\mathcal{B}(x, t)$ can be thought of as one of these observables capable of triggering a measurement process because it is one of the variables describing a system in interaction with the rest of the apparatus, with the position of the pointer in particular. In a quantum-theory formalism that is not translation invariant, $\mathcal{B}(x, t)$ corresponds to an operator $B(x)$ and, in our model, to a translation-invariant operator $\underline{B}$, independent of $x$. Let $\rho_{B}(x)$ be the nontranslation-invariant Fock-space operator associated with baryonic density at the point of coordinates $x$.

$$
\begin{gathered}
B(x)=\int_{\Omega(x)} \rho_{B}(x) d x^{3} \\
\underline{B}=e^{i P_{x}} B(x) e^{-i P_{x}}=\int_{\underline{\Omega}} \rho_{B}(x) d x^{3}=\int_{\underline{\Omega}} e^{-i P x} \rho_{B}(0) e^{i P x} d x^{3}
\end{gathered}
$$

where $\underline{\Omega}$ stands for $\Omega(0)$. 
The baryon number $\mathcal{B}(x, t)$ in the volume $\Omega(x)$ has a well-defined physical value wheneve' it is measured. At all times $t$, it has an expectation value referring to a possible and hypothetical measurement performed at time $t$.

$$
<\mathcal{B}(x, t)>=\operatorname{Tr}\left[B(x) D_{u}\right]=\operatorname{Tr}[\underline{B} \underline{D}(x, t)]
$$

In the model we define the quantity $\overline{\mathcal{B}}(x, t)$

$$
\overline{\mathcal{B}}(x, t)=\operatorname{Tr}[\underline{B} Q(x, t)]
$$

$\vec{B}(x, t)$ depends on the quantum-state matrix $\underline{Q}(x, t)$, which we use to represent reality at point $X$ of coordinates $x$ at time $t$ and on the Fock space operator $B$, which we define to be the same for all points in space; $\overline{\mathcal{B}}(x, t)$ is one of these real and local quantities attached to the point of coordinates $x$, i.e., depending only on real and loca! quantities. It is defined everywhere, every time, since $Q(x, t)$ is defined for all $x$ 's and $t$ 's.

Using Eq. (3.3), we can see that the expectation value $\langle\mathcal{B}(x, t)\rangle$ for the baryonic number in the small volume is related to the quantity $\overrightarrow{\mathcal{B}}(x, t)$, and the uncertainties $w_{k}$ about what the quantum-state matrix $Q(x, t)$ really is

$$
<\mathcal{B}(x, t)\rangle=\sum_{k} w_{k} \operatorname{Tr}\left[\underline{B} \underline{Q}_{k}(x, t)\right]=\sum_{k} w_{k} \overline{\mathcal{B}}_{k}(x, t)
$$

The function $\overline{\mathcal{B}}(x, t)$ is the expectation value of the baryonic charge $\mathcal{B}(x, t)$ in the small volume $\Omega(x)$ if it were measured at time $t$ and if there were no uncertainty about the matrix $Q(x, t)$.

In Eq. (5.1), instead of the baryonic density $\rho_{B}(x)$, one may use an altered operator $\rho_{B}^{\prime}(x)$ with the high-frequency components damped in momeatum space. This substitution may help reduce excessive heating effects during measurements if operator $B$ is chosen as the measurement operator $M$. The behavior of $B$ in Lorentz transformations has to be considered tco.

\subsection{Measurements Equated to Acts of Consciousness}

A quantum-theory description of the entire measurement process with an observer present involves interactions among

a) the quantum system 
b) the classical world

c) one or more observers.

Interactions between the quantum system and the classical world result in a change in the measuring apparatus located in the classical world (positioning of the pointer) and in the collapse of the wave function describing the quantum system. Interactions between the classical world and the observers involve biological phenomena and many unknown processes that we cannot specify. At least to some extent the dividing line between the quantum system and the classical world is arbitrary. ${ }^{\left[{ }^{[0]}\left[{ }^{[14]}\right.\right.}$ It has been suggested to move this line all the way toward the observer so that the collapse phenomenon becomes a phenomenon that only consciousness can produce. ${ }^{[15]} \mathrm{A}$ "measurement" is then equated with an act of consciousness. We first consider how this hypothesis could be made compatible with our model. In Section 5.3 we will consider another approach that separates reality from the phenomenon of consciousness.

Consciousness is an attribute of living humans. There is enough similarity in behavior between large animals and humans to make us assume that large animals have consciousness too. Analogy of behavior with humans is not a necessary requirement of consciousness, which, then, could be far more common in the universe than humans and large animals. Furthermore, the only property we are concerned with here is the ability to induce phenomena equivalent to collapses in quantum theory. This property alone may be even far more common than consciousness though not necessarily a property of ordinary matter.

In Section 5.2 we will give collapses the properties expected from them if they are phenomena caused by consciousness. Because the phenomena linking consciousness to the rest of the world are mostly unknown, we do not know the exact mathematical form of the measurement operator. We will just assume that it exists. In the model, the locations of the measurements, i.e., the points $m$ of coordinates $x_{m}$, coincide with the locations one could attribute to consciousness, i.e., with some points in space that we will call points of consciousness. As time evolves, each point of consciousness $m$ evolves along a trajectory $x_{m}(t)$, and "measurements," i.e, acts of consciousness are performed at times $t_{m}$ along the trajectory. These times $t_{m}$ are separated by finite time intervals so that the model is not plagued by the so-called Zeno's paradox.[16] 
An equation can be written for the trajectory of these so-called "points of consciousness." It is reasonable to make it coincide with the trajectory of a piece of matter. This can be done using the quantity $\mathcal{B}(x, t)$ of Eq. (5.4) as a guide.

$$
\frac{d x_{m}}{d t}=\zeta \frac{\partial \overrightarrow{\mathcal{B}}}{\partial x}
$$

where $\zeta$, like $\Omega$ in the definition (5.2) of $\underline{B}$, is a characteristic parameter of the trajectory. There is a large range of possible values for $\zeta$ and sizes for $\Omega$. Equation (5.6) involves only real and local quantities and thus can be called realistic and local. It has the property of making the trajectory point in the direction of a larger expectation value $\bar{B}$ of the baryonic charge density. Eventually, the trajectory will stay close to the line defined by the maximum of that expectation value, thus a line that could be chosen to define a trajectory for nearby nuclei. This property makes consciousness move in space with the matter constituting the.living bodies.

This approach to measurement theory is consistent with points of view that have been expressed before about quantum theory ${ }^{[8],[13]}$ and according to which Eq. (2.24) describes the evolution of all isolated physical systems not including elements of consciousness. However, it is not complete since a measurement operator cannot be defined due to our lack of knowledge of the phenomena involved in consciousness.

\subsection{The Spontaneous Measurements Approach}

For a complete description of the collapse phenomenon, it is better to leave consciousness and all the unknown phenomena surrounding it out of the model of objective reality. For this different approach one has to assume that "collapse phenomena" are spontaneous phenomena that can occur with the same properties as measurements in quantum theory but without the intervention of human observers or other censcious beings. This approach assumes that there are isolated physical systems that do not evolve according to Eqs. (2.24) or (3.9). Evolutions such as those described in Section 3.5 would apply instead. Therefore, in principle, there are deviations from quantum theory that may turn out to be detectable experimentally, ${ }^{[0]}$ However, if these collapses have significant effects only 
when macroscopic objects are present, these deviations from quantum theory may have been invisible so far. Spontaneous collapses have been suggested before ${ }^{[17]}$ but without the locality imposed on the model described in this paper. In Section 5.3 we will describe a possible framework for spontaneous measurements in the context of this model. We will keep using the word "measurement" for the processes that induce these collapses, even though no human is necessarily present to observe the result.

Measurements no longer occur along trajectories determined by an equation like Eq. (5.6). However, in a model emphasizing locality, the location of the measurement is still crucial. We will assume that measurements can happen at any point in space and that their statistical behavior is ruled by a Poisson process. Given a small volume $d v$ around a point of coordinates $x$ and a time interval $d t$ there is a probability $\Gamma(x, t) d v d t$ that a "measurement" followed by a collapse occurs at a point $m$ of coordinates $x_{m}$ anywhere in the volume $d v$ at any time $t_{m}$ in the interval $d t$. The probability distribution $\Gamma(x, t)$ can be made uniform in space and time

$$
\Gamma(x, t)=\text { constant over the universe. }
$$

or it is possible to make $\Gamma(x, t)$ dependent on real and local quantities defined using the matrix $Q(x, t)$. For instance, $\Gamma(x, t)$ could be dependent on the expectation values $\overline{\mathcal{B}}_{j}(x, t)$ of the number of baryons $\mathcal{B}_{j}(x, t)$ in small volumes $\Omega_{j}(x)$ surrounding point $X$ of coordinates $x$. If the $\Omega_{j}(x)$ are of different sizes, the rate $\Gamma(x, t)$ depends on the structure of matter surrourding point $X$.

A measurement operator, $M$, must be chosen. It must be a translationinvariant operator and a function of the field operators $\varphi_{j}(x)$ in Fock space, as expressed by Eqs. (2.10), (2.12), and (2.16); $M$ can be made independent of the location of point $m$ if one assumes that the function $f_{j}(x)$ in Eq. (2.12) is actually a function $f_{j}\left(x-x_{m}\right)$ of $x-x_{m}$. Then, the general form for $\underline{M}$ is

$$
\begin{aligned}
& \underline{M}=\underline{M}\left(\underline{\phi}_{j}\right) \\
& \underline{\phi}_{j}=\int \underline{f}_{j}(\Delta x) F_{j}\left[\varphi_{j^{\prime}}(\Delta x)\right] d x^{3}
\end{aligned}
$$

where the integral in Eq. (5.9) extends over a volume of size $L_{m}$, which, as 
pointed out in Section 5.1, we prefer to take small, if possible of the order of the atomic scale, around the origin of the space coordinates.

In. the framework of the model, several observables $\mathcal{M}$, i.e., several operators $M$ with properties (5.8) and (5.9), can be conceived. Each of these operators $\underline{M}$ has eigenvectors that determine a basis in which density matrices get diagonalized in the measurement process. This basis has been called the "pointer basis." [18] For each basis there is a different measurement theory. The theory will be successful if the corresponding observable $\mathcal{M}$ is correlated enough with the other variables of the apparatus to explain collapses during measurements but does not disturb isolated quantum systems significantly. Adjustment of key parameters and comparison with practical applications are necessary. It is beyond the scope of this paper to analyze all the possibilities and then make a choice of observables $\mathcal{M}$ for the spontaneous measurements. Such analysis will be the subject of future papers.

\section{CONCLUSION}

It has been possible to construct a realistic model that satisfies a rudimentary locality property and, to an arbitrary degree of accuracy, reproduces the predictions of relativistic quantum theory. The model is realistic because it uses physical quantities $Q(x, t), C(x, t)$, and $\underline{M}$ that exist independently of an observer's knowledge. The model is translation invariant and local in a rudimentary sense because it has the following properties:

a) $\underline{Q}(x, t)$ and $C(x, t)$ are defined as quantities attached to a point $X$ in space with coordinates $x$;

b) the operator $M$ is attached to a point $m$ of coordinates $x_{m}$ where the measurement is performed;

c) all these quantities are invariant when the origin of the space coordinates is changed;

d) the evolutions of $Q(x, t)$ and $C(x, t)$ are described by differential equations in $x$ and $t$ (Eqs. (3.4) in Section 3 and (A.17) in Appendix A). There is no instantaneous action at distance. 
In the model, there is a privileged space-time restframe and a parameter $V$ with the dimension of a velocity; $V$ is assumed to be much larger than c. If a model of this type is correct, no violation of the predictions of quantum theory is expected if time intervals between measurements are large, i.e., larger than the time necessary to travel between the locations of these measurements at the velocity $V>c$ in the privileged restframe. In particular, no identification of the privileged restframe would be possible using such measurements. However, by performing multiple-measurement experiments with large distances $\Delta x$ and time intervals smaller than $\Delta x / V$ between measurements, violations of quantum theory may be expected. Some identification of the privileged restframe may become possible.

If the value of parameter $V$ is very high, the time interval $\Delta x / V$ is so small that the multiple-measurement experiments mentioned above become impractical. As long as this is true, the model will show no observable discrepancy with quantum theory. Its impact will be primarily philosophical.

If parameter $V$ is not too large, multiple-measurement experiments of the type described above may reveal a violation of quantum theory and of Lorentz invariance at the same time. Whenever such experiments uphold the predictions of quantum theory, they allow one to derive a lower limit for the parameter $V$.

In the framework of the model, it is possible to develop local measurement theories. To be completely satisfactory, they require more analysis "han was possible in this paper.

\section{ACKNOWLEDGEMENTS}

I am deeply indebted to Dr. H.P. Stapp for a critical review of this paper and fruitful discussions about it. 


\section{REFERENCES}

[1] See, for instance, M. Jammer, The Philosophy of Quantum Mechanics (Wiley, New York, 1974), or E.P. Wigner in Quantum Theory and Measurement (Princeton University Press, Princeton, NJ, 1983).

[2] For a general review, see F.J. Belinfante, A Survey of Hidden Variables Theories (Pergamon Presș, New York, 1973). See also, for instance, P. Droz-Vincent, Phys. Rev. D 19 (1979) 702 and J.-P. Vigier, Lett. Nuovo Cim. 24 (1979) 258.

[3] D. Bohm, Wholeness and the Implicate Order (Routledge and Kegan Paul, London, 1980) Chap. 4;

D. Bohm and B. Hiley, Found. Phys. 5 (1975) 93.

[4] J.S. Bell, Physics 1 (1964) 195.

[5] Generalizations of Bell's Theorem have been attempted:

J.F. Clauser, M.A. Horne, A. Shimony and R.A. Holt, Phys. Rev. Lett. 23 (1969) 80;

H.P. Stapp, Phys. Rev. D 3 (1971) 1303;

J.F. Clauser and M.A. Horne, Phys. Rev. D. 10 (1974) 526;

J.S. Bell, CERN preprint Th 2053, reproduced in Epistem. Lett. (Assoc. F. Gonseth CP 1081, CH-205 Bienne) 9 (1975) 11;

H.P. Stapp, Nuovo Cim. 40B (1977) 191;

P.H. Eberhard, Nuovo Cim. 46B (1978) 392;

H.P. Stapp, Quantum Nonlocality, LBL-22815 (1987).

(6) A. Einstein, B. Podolsky and N. Rosen, Phys. Rev, 47 (1935) 777.

[7] See, for instance, S.S. Schweber, Relativistic Quantum Field Theory, (Row, Peterson and Co, Evanston, IL, 1961), which we used as a reference.

[8] J. von Neumann, Mathematical Foundations of the Quantum Mechan. ics (Springer-Verlag, Berlin, 1932). (Also reprinted in translation by Princeton University Press, Princeton, NJ, 1955.)

[9] This point was stressed in P.H. Eberhard, CERN preprint CERN 72-1 (1972).

[10] See, for instance, W.C. Carithers et al., Phys. Rev. D 14 (1976) 290; P.H. Eberhard et al., Phys. Lett. B 53 (1974) 121. 
[11] By the generic name, Bell's inequalities, this paper refers to all the inequalities based on a locality condition and expressed in different forms by different authors. See, in particular:

J.S. Bell, Physics 1 (1964) 195;

J.F. Clauser, M.A. Home, A. Shimony and R.A. Holt, Phys. Rev. Lett. 23 (1969) 880.

[12] B. d'Espagnat, Conceptual Foundations of Quantum Mechanics (W.A. Benjamin, Reading, MA, 1976) Part 4, p. 58 ff.

[13] See, for instance, P.H. Eberhard, Nuovo Cim. 46B (1978) 392 (APpendix D).

[14] For a general survey of measurement theory, see M. Jammer, Conceptual Development of Quantum Mechanics (McGraw-Hill, New York, 1966);

B. d'Espagnat, Conceptual Foundations of Quantum Mechanics (2nd Edition) (Benjamin, New York [now Addison-Wesley, Reading, MA], 1976);

J.A. Wheeler and W. Zurek, Eds Quantum Theory and Measurement (Princeton University Press, Princeton, NJ, 1983).

[15] E.P. Wigner, in The Scientist Speculates (1982) reprinted in Quantum Theory and Measurement eds. J.A. Wheeler and W: Zurek (Princeton University Press, Princeton, NJ, 1983).

(16] B. Misra and E.C.G. Sudarshan, J. Math. Phys. 18 (1977) 756;

C.B. Chiu, E.C. G. Sudarshan and B. Misra, Phys. Rev. D 16 (1977) 520.

[17] G.C. Ghirardi, A. Rimini and T. Weber, Phys. Rev. D 34 (1986) 470 .

[18] W.H. Zurek, Phys. Rev, D 24 (1981) 1516. 


\section{APPENDIX A: A Partial Derivative Equation for $C(x, t)$}

To get a differential equation for the evolution of $C(x, t)$ of Eq. (3.6) we first consider an equation that would be satisfied by any function $F\left(t-T(x)-t_{m}\right)$ of $t-T(x)-t_{m}$, where $T(x)$ is defined by Eq. (3.5), i.e.,

$$
T(x)=\frac{\left|x-x_{m}\right|}{V}
$$

We have the following relations:

$$
\begin{aligned}
\frac{\partial T}{\partial x} & =\frac{x-x_{m}}{V^{2} T} \\
\nabla^{2} T & =\frac{2}{V^{2} T} \\
\nabla^{2}\left(\frac{1}{T}\right) & =-4 \pi V \delta\left(x-x_{m}\right)
\end{aligned}
$$

where the symbol $\nabla^{2}$ stands for the Laplacian in three-dimensional space. Let us introduce the symbol $\diamond$ for an operator similar to the Dalembertian but where the velocity of light $c$ has been replaced by the velocity $V$

$$
\diamond=\frac{1}{V^{2}} \frac{\partial^{2}}{\partial t^{2}}-\nabla^{2}
$$

Then

$$
\diamond F=\frac{2}{V^{2} T} \frac{\partial F}{\partial t}
$$

$\frac{\partial F}{\partial t}$ is also a function of $t-T(x)-t_{m}$. Therefore,

$$
\begin{aligned}
\infty & =\frac{2}{V^{2}}\left[\frac{\partial F}{\partial t} \diamond\left(\frac{1}{T}\right)-2 \frac{\partial\left(\frac{1}{T}\right)}{\partial x} \frac{\partial^{2} F}{\partial x \partial t}+\frac{1}{T} \diamond \frac{\partial F}{\partial t}\right] \\
& =\frac{2}{V^{2}}\left[-\frac{\partial F}{\partial t} \nabla^{2}\left(\frac{1}{T}\right)-\frac{2}{T^{2}} \frac{\partial^{2} F}{\partial t^{2}}\left|\frac{\partial T}{\partial x}\right|^{2}+\frac{2}{V^{2} T^{2}} \frac{\partial^{2} F}{\partial t^{2}}\right] \\
& =\frac{8 \pi}{V} \frac{\partial F}{\partial t} \delta\left(x-x_{m}\right)
\end{aligned}
$$


The operator $C(x, t)$, which is given by Eq. (3.6), is not a function of $t-T(x)-t_{m}$ because $\pi(x, t)$ has the space and time dependence shown in Eq. (3.7). However, we can define another operator, $C(x, t)$, that is a function of $t-T(x)-t_{m}$ and, therefore, that obeys Eq. (A.7)

$$
\begin{aligned}
C(x, t) & =e^{i H\left(t-t_{m}\right)} e^{-i P\left(x-x_{m}\right)} C(x, t) e^{i P\left(x-x_{m}\right)} e^{-i H\left(t-t_{m}\right)} \\
& =\lambda_{m} \Pi_{\mu}\left[\Theta\left(t-T(x)-t_{m}\right)-\Theta\left(t-T(x)-\tau_{m}-t_{m}\right)\right](A \\
\diamond \diamond C(x, t) & =\frac{8 \pi \lambda_{m}}{V} \underline{\Pi}_{\mu}\left[\delta\left(t-t_{m}\right)-\delta\left(t-\tau_{m}-t_{m}\right)\right] \delta\left(x-x_{m}\right)
\end{aligned}
$$

In Eq. (A.9), $C(x, t)$ can be replaced by its first expression in Eq. (A.8). For convenience, let us define operators $\underline{\partial}_{t}$ and $\nabla_{t}$ for Fock-space operators $\mathcal{O}(x, t)$ with an $x t$ space-time dependence; $\underline{\partial}_{r}$ and $\underline{\nabla_{s}}$ are defined so that

$$
\begin{aligned}
\underline{\partial}_{f} \mathcal{O} & =\frac{\partial \mathcal{O}}{\partial t}+i(H \mathcal{O}-\mathcal{O} H) \\
\underline{\nabla}_{x} \mathcal{O} & =\frac{\partial \mathcal{O}}{\partial x}-i(P \mathcal{O}-\mathcal{O} P)
\end{aligned}
$$

These operators have the property that

$$
\begin{aligned}
\frac{\partial}{\partial t}\left[e^{i H\left(t-t_{m}\right)} \mathcal{O} e^{-i H\left(t-t_{m}\right)}\right] & =e^{i H\left(t-t_{m}\right)}\left(\underline{\partial}_{f} \mathcal{O}\right) e^{-i H\left(t-t_{m}\right)} \\
\frac{\partial}{\partial x}\left[e^{-i P\left(x-x_{m}\right)} \mathcal{O} e^{i P\left(x-x_{m}\right)}\right] & =e^{-i P\left(x-x_{m}\right)}\left(\underline{\nabla}_{x} \mathcal{O}\right) e^{i P\left(x-x_{m}\right)}
\end{aligned}
$$

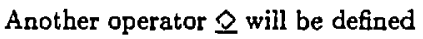

$$
\underline{\imath}=\frac{1}{V^{2}}\left(\underline{\partial}_{t}\right)^{2}-\underline{\nabla}_{x} \cdot \underline{\nabla}_{x}
$$

It has a property derived from Eggs. (A.5), (A.12), and (A.13)

$$
\begin{aligned}
& \diamond\left[\left(e^{i H\left(t-t_{m}\right)} e^{-i P\left(x-x_{m}\right)} \mathcal{O}(x, t) e^{i P\left(x-x_{m}\right)} e^{-i H\left(t-t_{m}\right)}\right]\right. \\
& =e^{i H\left(x-t_{m}\right)} e^{-i P\left(x-x_{m}\right)}(\underline{O}) e^{i P\left(x-x_{m}\right)} e^{-i H\left(t-t_{m}\right)}
\end{aligned}
$$

Therefore, using Eq. (A.7),

$$
\diamond \diamond C(x, t)=e^{i H\left(t-t_{m}\right)} e^{-i P\left(x-x_{m}\right)}[\underline{\varnothing} \underline{\otimes C}(x, t)] e^{i P\left(x-x_{m}\right)} e^{-i H\left(t-t_{m}\right)}
$$


Therefore, Eq. (C.9) can be written as an equation for $C(x, t)$

$\underline{\otimes} \underline{\varnothing C}(x, t)=\frac{4 \pi \lambda_{m}}{V}\left[\underline{\Pi}_{\mu} \delta\left(t-t_{m}\right)-e^{-i H \tau_{m}} \underline{\Pi}_{\mu} e^{i H \tau_{m}} \delta\left(t-\tau_{m}-t_{m}\right)\right] \delta\left(x-x_{m}\right)$

Equation (A.17) is of a fourth order in $t$. It unambiguously determines the operator $C(x, t)$ as the quantity given in Eq. (3.6) when Eq. (A.17) is complemented by the conditions $C(x, t) \equiv 0$ for some time interval in the past and for all $x$ 's. Furthermore, operators $\underline{\partial}_{t}, \underline{\nabla}_{x}$, and therefore $\underline{\imath}$ generate operators defined at point $x$ and $t$ using operators defined at that point and in its neighborhood only. They are local. $I_{\mu}$ is an operator defined at point $m$ only, but, in Eq. (A.17), it is multiplied by the Dirac distribution $\delta\left(x-x_{m}\right)$. It plays a role only at the point $m$, such as $x=x_{m}$. Therefore, Eq. (A.17) is local.

If more than one measurement is performed, we assume $C(x, t)$ stil] to be determined by Eq. (A.17). Equation (A.17) is linear. Therefore, operator $C$ will be the sum of the contributions computed for each measurement separately.

\section{APPENDIX B: Conservation of the Spatial-Compatibility Condition}

The spatial-compatibility condition (3.20) can be rewritten using the matrix $\tilde{Q}(x, t)$, which is not translation invariant and which is defined as

$$
\tilde{Q}(x, t)=e^{i H t} e^{-i P x} Q(x, t) e^{i P x} e^{-i H t}
$$

The spatial-compatibility condition can be expressed as

$$
\tilde{Q}\left(x_{1}, t\right) \cong \tilde{Q}\left(x_{2}, t\right)
$$

During a transition-free period, i.e., when the collapse operator is zero, the quantum matrix $\underline{Q}(x, t)$ obeys Eq. (3.8); therefore $\tilde{Q}(x, t)$ is a constant

$$
\frac{\partial \tilde{Q}(x, t)}{\partial t}=0
$$


Therefore, if Eq. (B.2) holds at the beginning of a transition-free period it also holds at the end of that period.

After a transition period generated by a measurement giving the result $\mathcal{M}=\mu, \underline{Q}\left(x, t_{f}\right)$ is given by Eq. (3.15). From that equation we can derive an expression for $\tilde{Q}\left(x, t_{f}\right)$ defined from $\underline{Q}\left(x, t_{f}\right)$ by Eq. (B.1). We first define the operator

$$
\begin{aligned}
\Lambda_{\mu} & =e^{i H t_{m}} e^{-i P_{x}} e^{\pi_{\mu}(x) \lambda_{m} \tau_{m}} e^{i P_{x}} e^{-i H t_{m}} \\
& =e^{i H t_{m}} e^{I_{\mu} \lambda_{m} \tau_{m}} e^{-i H t_{m}}
\end{aligned}
$$

using the definition (2.21) of $\underline{I}_{\mu}(x)$ and (2.17) of $\Pi_{\mu}$. Then, Eq. (3.15) becomes

$$
\tilde{Q}\left(x, t_{f}\right)=\frac{1}{\mathcal{N}\left(t_{f}\right)} \Lambda_{\mu} \tilde{Q}\left(x, t_{m}\right) \Lambda_{\mu}^{+}
$$

As shown by Eq. (B.3), $\tilde{Q}(x, t)$ is constant outside of the transition period, i.e., for $t>t_{f}$ and $t<t_{m}$. Therefore, given a time interval $t_{0} t$ in which only one transition period due to one measurement occurs, we have a relation between the matrix $\tilde{Q}(x, t)$ and $\tilde{Q}\left(x, t_{0}\right)$ for all points $x$ such that $t_{f}<t$

$$
\tilde{Q}(x, t)=\frac{1}{\mathcal{N}\left(t_{f}\right)} \Lambda_{\mu} \tilde{Q}\left(x, t_{0}\right) \Lambda_{\mu}^{+}
$$

The operator $\Pi_{\mu}$ depends on the point $m$ of measurement and on the choice of origin of the spatial coordinates. It does not depend on the variable $x$ figuring in the $\bar{Q}(x, t)$ 's of Eq. (B.5). Therefore, $\Lambda_{\mu}$ does not depend on $x$. Consider two points $x_{1}$ and $x_{2}$ in space. The same operator $\Lambda_{\mu}$ will affect the matrices $Q\left(x_{1}, t\right)$ and $Q\left(x_{2}, t\right)$ after a measurement at time $t_{m}$ and at spatial coordinates $x_{m}$, though possibly at a different time due to possible different time of propagation $T\left(x_{1}\right)$ and $T\left(x_{2}\right)$ of the transition period to the two points. Consider a time $t_{0}$ that precedes either the measurement time $t_{m}$ or, at least, both times $t_{m}+T\left(x_{1}\right)$ and $t_{m}+T\left(x_{2}\right)$ at which the transition period reaches $x_{1}$ and $x_{2}$. Consider another time, $t>t_{m}$, after both transition periods have ended in $x_{1}$ and $x_{2}$. The evolutions of $\tilde{Q}\left(x_{1}, t\right)$ and $\tilde{Q}\left(x_{2}, t\right)$ satisfy Eq. (B.6) with the same operator, $\Lambda_{\mu}$.

It follows that, if Eq. (B.2) is satisfied at time $t_{0}$, it is satisfied also at time $t$. Since Eq. (B.2) is equivalent to the spatial-compatibility condition (3.20), one concludes that the spatial compatibility, if valid initially, 
will stay valid at some times later. This is true whether or not measurements are being performed as long as each of the transitions induced by all measurements has reached either all or none of the points in space under consideration.

\section{APPENDIX C: Generating the Spatial-Compatibility Condition}

At some initial time $t_{0}$ we assume that the matrices $\underline{Q}\left(x, t_{0}\right)$ do not satisfy Eq. (3.20). We only assume that these matrices are of a finite rank $N$, the same for all matrices $Q\left(x, t_{0}\right)$ at all $x$ 's and with some additional conditions as to the space spanned by their eigenvectors. Then many events occur, generating collapses as in Eq. (3.15) in conditions such that the rank is reduced. We will prove that this process generates the spatialcompatibility condition (3.20).

We will use the formalism developed in Appendix B. The spatial-compatibility condition we want to generate will be expressed as in Eq. (B.2), instead of Eq. (3.20), which is equivalent. We will use the matrices $\tilde{Q}(x, t)$ defined by Eq. (B.1). The rank of the matrices $\tilde{Q}\left(x, t_{0}\right)$ is the same as the rank of the matrices $\underline{Q}\left(x, t_{0}\right)$, thus finite.

$$
\tilde{Q}\left(x, t_{0}\right)=\sum_{k=1}^{N} q_{0 k}(x) \Psi_{0 k}(x) \Psi_{0 k}^{+}(x)
$$

To avoid having to deal with pathological cases we will assume that the matrices $\tilde{Q}\left(x, t_{0}\right)$ for all $x$ 's are of the same rank $N$ and that their eigenvectors for nonzero eigenvalues, $\Psi_{0 k}(x)$, define the same subspace, i.e., a subspace independent of $x$. Therefore, whatever $x_{1}$ and $x_{2}$ are, each one of the eigenvectors for nonzero eigenvalue $\Psi_{0 k}\left(x_{2}\right)$ of $\tilde{Q}\left(x_{2}, t_{0}\right)$ is a linear combination of the eigenvectors for nonzero eigenvalues $\Psi_{0 k}\left(x_{1}\right)$ of $\tilde{Q}\left(x_{1}, t_{0}\right)$.

When an event occurs with the same effects as a measurement, the matrices $\tilde{Q}(x, t)$ collapse os in Eq. (B.6), with the operator $\Lambda_{\mu}$ defined by Eq. (B.4). We now define another projection operator $\tilde{\Pi}_{\mu}$ associated with the outcome $\mu$ of the measurement of $\mathcal{M}$ at time $t_{m} ; \tilde{\Pi}_{\mu}$ is the projection operator for such an outcome in the Heisenberg representation. It is 
independent of the coordinates $x$

$$
\begin{aligned}
\check{\Pi}_{\mu} & =e^{i H t_{m}} e^{-i P z_{m}} \underline{\Pi}_{\mu} e^{i P x_{m}} e^{-i H t_{m}} \\
& =e^{i H t_{m}} \Pi_{\mu} e^{-i H t_{m}} \\
\Lambda_{\mu} & =e^{\Lambda_{\mu} \lambda_{n_{3}} \tau_{m}}=\tilde{\Pi}_{\mu}\left(e^{\left.\lambda_{m} t_{m}\right)}-1\right)+I
\end{aligned}
$$

We first consider the limit $\lambda_{m} \tau_{m}=\infty$. Then, taking Eq. (C.1) into account, Eq. (B.6) for $t$ larger than $t$, of Eq. (3.14) becomes

$$
\tilde{Q}(x, t)=\frac{1}{\mathcal{N}} \sum_{k=1}^{N} q_{o k}(x) \Psi_{f k}(x) \Psi_{f k}^{+}(x)
$$

where

$$
\Psi_{f k}(x)=\tilde{\Pi}_{\mu} \Psi_{0 k}(x)
$$

Since the summation over $k$ in Eq. (C.4) extends to a number of terms $N$ equal to the number of terms in Eq. (C.1.), the rank of the matrix $\tilde{Q}(x, t)$ after measurement cannot be higher than the rank before. However, it can be less. Suppose for a given space coordinate $x_{1}$ there is a linear combination of the $\Psi_{0 k}\left(x_{1}\right)$

$$
\Psi=\sum_{k} c_{k} \Psi_{0 k}\left(x_{1}\right)
$$

such that

$$
\tilde{\Pi}_{\mu} \Psi=0
$$

The projection operator $\tilde{\Pi}_{\mu}$ is linear. Using Eqs. (C.6) and (C.5), it is easy to show that Eq. (C.7) expresses a linear relationship between the $\Psi_{f k}\left(x_{1}\right)$

$$
\sum_{k} c_{k} \Psi_{f k}\left(x_{1}\right)=0
$$

Under such circumstances there are no more than $N-1$ linear independent vectors among the $\Psi_{J k}$ 's. Therefore, the matrix $\bar{Q}\left(x_{1}, t\right)$ of Eq. $(\mathrm{C.} 4)$ is at most of rank $N-1$.

Now we will show that, fur times $t$ larger than the values of $t_{f}$ defined by Eq. (3.14) at both points of coordinates $x_{1}$ and $x_{2}$, the matrix $\dot{Q}\left(x_{2}, t\right)$ for any $x_{2} \neq x_{1}$ is reduced also to the same rank as the rank of $\bar{Q}\left(x_{1}, t\right)$. 
This property results from our hypothesis that any one of the $\Psi_{0 k}\left(x_{1}\right)$ is a linear combination of the $\Psi_{0 k}\left(x_{2}\right)$.

$$
\Psi_{0 k}\left(x_{1}\right)=\sum_{k^{\prime}} c_{k k^{\prime}} \Psi_{0 k^{\prime}}\left(x_{2}\right)
$$

Because of the linearity of the operator $\tilde{\Pi}_{\mu}$ used in Eq. (C.5), any $\Psi_{f k}\left(x_{1}\right)$ can be expressed by the same linear combination of the $\Psi_{f k}\left(x_{2}\right)$ with the same coefficients.

$$
\Psi_{f k}\left(x_{1}\right)=\tilde{\Pi}_{\mu} \sum_{k^{\prime}} c_{k k^{\prime}} \Psi_{0 k^{\prime}}\left(x_{2}\right)=\sum_{k^{\prime}} c_{k k^{\prime}} \Psi_{f k^{\prime}}\left(x_{2}\right)
$$

Therefore, the $\Psi_{f k}\left(x_{1}\right)$ cannot define a space with more dimensions than the space of the $\Psi_{f k}\left(x_{2}\right)$. The space of the $\Psi_{f k}\left(x_{1}\right)$ is identical to the space of the $\Psi_{f k}\left(x_{2}\right)$ or is a subspace of it. The same argument can be developed interchanging $x_{1}$ and $x_{2}$. Therefore, the $\Psi_{f k}(x)$ define the same space for all $x$ 's, and the $\tilde{Q}(x, t)$ have the same rank.

We can also show that the rank of all the $\bar{Q}^{\prime} s$ will never be reduced below rank 1. If it were, the quantum matrix $\bar{Q}\left(x_{m}, t\right)$ of Eq. (C.4) at the measurement point $x_{m}$ would be zero. Therefore, because of Eqs. (3.1), (B.1) and (C.2)

$$
p_{\mu}=\operatorname{Tr}\left[\underline{\Pi}_{\mu} \underline{Q}\left(x_{m}, t_{m}-\epsilon\right)\right]=\operatorname{Tr}\left[\tilde{\Pi}_{\mu} \tilde{Q}\left(x_{m}, t_{0}\right)\right]=0
$$

The probability of such outcome $\mu$ is zero. Therefore, the measurement will have to give another outcome for which the probability is not zero. The projection operator cannot zeduce the rank of the matrices $\tilde{Q}(x, t)$ to zero.

We now consider the case of a large number of events generating collapses as in measurements performed in a universe initially made of matrices $Q\left(x, t_{0}\right)$ that do not satisfy the compatibility condition. In addition to the assumptions made above with regard to the rank of the matrices $\tilde{Q}\left(x, t_{0}\right)$ and the subspace defined by their eigenvectors, we now hypothesize that, among this large number of measurements, many outcomes correspond to projection operators $\tilde{\Pi}_{\mu}$ that reduce the rank of the matrices $\tilde{Q}$ by the mechanism described above. We assume that the rank-reducing processes went so far as to reduce the rank of all the matrices $\tilde{Q}(x, t)$ to the rank 1 . Since their eigenvectors define the same space and since their trace is one, 
the matrices $\tilde{Q}(x, t)$ are the same for all $x$ 's. Equation (B.2), and therefore Eq. (3.20), has been made to be true with $100 \%$ accuracy.

In all this development since Eq. (C.4) we have considered the limit $\lambda_{m} \tau_{m}=\infty$ in Eq. (C.3). That limit corresponds to an approximation. When the effect of the identity matrix in Eq. (C.3) is taken into account, it is easy to see that the ranks are reduced only for matrices that represent an approximation for the matrices $\tilde{Q}$, not for the real ones. There is a small correction in $\tilde{Q}(x, t)$, and that correction depends on the initial value $\tilde{Q}\left(x, t_{0}\right)$. Therefore, the final matrices $\tilde{Q}(x, t)$ are only approximately equal to matrices of rank 1 , and the spatial-compatibility condition holds only approximately.

In conclusion, we have found a way for the spatial-compatibility condition of Eq. (3.20) to be generated in a universe for which that condition was not true initially. What is needed are many events generating collapses as in Eq. (B.6), i.e., as in Eq. (3.15), which is equivalent. Such events could be the spontaneous measurements described in Section 5.3.

\section{APPENDIX D: Collapses Due to Two Measurements Closely Spaced in Time}

Whenever two measurements are performed at $m_{1}$ and $m_{2}$ at time $t_{m l}$ and $t_{m 2}>t_{m 1}$, respectively, two transition zones propagate away from the points of measurement, one from $m_{1}$ and one from $m_{2}$. They reach an arbitrary point of coordinates $x$ in space at times $t_{x 1}$ and $t_{x 2}$, respectively. This is a phenomenon described in Section 4.3 in the context of measurements well spaced in time. For measurements closely spaced in time, i.e., if the spatial separation $\Delta x$ between $m_{1}$ and $m_{2}$ and the time interval $\Delta t$ between $t_{m 1}$ and $t_{m 2}$ satisfy inequality (4.14), there are points in space for which $t_{x 1}<t_{x 2}$ and other points for which $t_{x 2}<t_{x 1}$. Furthermore, the joint probability of the outcomes $\mu_{1}$ and $\mu_{2}$, thus of the projection operators $\underline{\Pi}_{\mu 1}$ and $\Pi_{\mu 2}$ is given by Eqs. (4.16), (4.17), and (4.15), which may be different from the predictions of quantum theory.

As in Appendices $B$ and $C$, we will describe the collapses using the operators $\tilde{Q}(x, t)$ defined by Eq. (B.1) and $\Lambda_{\mu}$ defined by Eq. (B.4) but 
where we take the limit $\lambda_{m} \tau_{m}=\infty$. Equation (B.6) becomes

$$
\tilde{Q}(x, t)=\frac{1}{\mathcal{N}} \ddot{\Pi}_{\mu} \tilde{Q}\left(x, t_{0}\right) \tilde{\Pi}_{\mu}
$$

where $\tilde{\Pi}_{\mu}$ is the projection operator defined by Eq. (C.2) corresponding to the outcome $\mu$ in the Heisenberg representation. For Eq. (D.1) to be valid, the time interval to $t$ contains one and only one transition period, i.e., either the time $t_{x 1}$ or the time $t_{x 2}$. If the interval $t_{0} t$ contains two transition periods, i.e., $t_{x 1}$ and $t_{x 2}$, Eq. (D.1) has to be applied twice. For the points of coordinates $x$ such that $t_{x 1}<t_{z 2}$,

$$
\tilde{Q}(x, t)=\frac{1}{\mathcal{N}} \tilde{\Pi}_{\mu 2} \tilde{\Pi}_{\mu 1} \tilde{Q}\left(x, t_{0}\right) \tilde{\Pi}_{\mu 1} \tilde{\Pi}_{\mu 2}
$$

For the points for which $t_{x 2}<t_{x 1}$

$$
\tilde{Q}(x, t)=\frac{1}{\overline{\mathcal{N}}} \tilde{\Pi}_{\mu 1} \tilde{\Pi}_{\mu 2} \tilde{Q}\left(x, t_{0}\right) \tilde{\Pi}_{\mu 2} \tilde{\Pi}_{\mu 1}
$$

Expressions (D.2) and (D.3) are identical if the operators $\tilde{\Pi}_{\mu 1}$ and $\tilde{\Pi}_{\mu 2}$ commute. We will show that, in almost all cases, they do commute for measurements closely spaced in time. Let us replace $\Pi_{\mu}$ in Eq. (C.2) by its expression (2.11), taking Eq. (2.12) into account

$$
\begin{gathered}
\tilde{\Pi}_{j}=\tilde{\Pi}_{\mu}\left(\tilde{\phi}_{j}\right) \\
\tilde{\phi}_{j}=\int f_{j}(x) F_{j}\left[\tilde{\varphi}_{j}\left(x, t_{m}\right)\right] d x^{3}
\end{gathered}
$$

where the integral extends over a range of $z$ 's of dimension $L_{m}$ representing the size of the apparatus.

$$
\tilde{\varphi}_{j}(x, t)=e^{i H t} \varphi_{j}(x) e^{-i H t}
$$

The operator $\tilde{\varphi}_{j}(x, t)$ is the field operator in the Heisenberg representation. In quantum field theory commutation and anti-commutation rules have been set so that $\tilde{\varphi}_{j}\left(x_{1}, t_{1}\right)$ and $\tilde{\varphi}_{j}\left(x_{2}, t_{2}\right)$ commute if

$$
\left|z_{2}-z_{1}\right|>c\left|t_{2}-t_{1}\right|
$$


For two measurements closely spaced in time, the distance $\Delta x$ between the two points $m_{1}$ and $m_{2}$, which lie inside of apparati 1 and 2, respectively, is larger than the product of the values of the parameter $V$ and the time interval $\Delta t$ as stated in inequality (4.14). Therefore, $\Delta x$ is much larger than the product $c \cdot \Delta t$. For points $m_{1}$ and $m_{2}$ at $t_{m 1}$ and $t_{m 2}$, inequality (D.7) is satisfied with a large margin. If a non-negligible fraction of this distance $\Delta x$ is made of a physical separation between apparati, any set of two points made of one point of coordinates $x_{1}$ in the first apparatus at time $t_{m 1}$ and one point of coordinates $x_{2}$ in the second apparatus at time $t_{m 2}$ also satisfies inequality (D.7). It follows that the operators $\tilde{\Pi}_{\mu 1}$ and $\tilde{\Pi}_{\mu 2}$ commute. Expression (D.2) can be used everywhere in space to express the collapse due to the two transition periods. If $\bar{Q}(x, t)$ is approximately independent of $x$ at time $t=t_{0}$ before the two measurements, it follows from the equivalence of Eqs. (D.2) and (D.3) that $\tilde{Q}(x, t)$ will have the same property any time later everywhere the two transition periods have been completed. Thus, the spatial-compatibility condition is also conserved by measurements closely spaced in time.

In the rare cases where the apparati are sitting next to one another but the measurements are still closely spaced in time, $\tilde{\Pi}_{\mu 1}$ and $\tilde{\Pi}_{\mu 2}$ may not commute. A small violation of the spatial-compatibility condition may be generated. This condition can most probably be restored later on by the kind of pheinomenon described in Appendix C. These rare effects may be studied more meaningfully within the framework of a complete measurement theory.

In any event, to make practical computations, note that Eq. (D.2) is independent of the parameter $V$ as long as $V$ keeps the inequalities $t_{0}<t_{x 1}<t$ and $t_{0}<t_{x 2}<t$ valid. It follows that after the two collapses the matrix $\tilde{Q}(x, t)$, and therefore the matrix $\underline{Q}(x, t)$, are the same as if $V$ were infinite and the outcomes of the messurements were still $\mu_{1}$ and $\mu_{2}$. The only difference is that, for measurements closely spaced in time, the joint probability $\rho_{\mu 1 \mu 2}$ distribution of $\mu_{1}$ and $\mu_{2}$ is given by Eqs. (4.16), (4.17), and (4.15). As mentioned in Section 4.4, it may differ from the predietions of the model when $V=\infty$, i.e., from the predictions of quantum theory.

The change in our uncertainties $w_{k}$ about the matrices $\underline{Q}_{f}(x, t)$ is expressed by a standard application of formulae for conditional probabilities 
as we did for Eq. (3.22).

$$
w_{k}(t)=\frac{w_{k}\left(t_{0}\right) \wp_{k \mu 1} \wp_{k \mu 2}}{\sum w_{k^{\prime}}\left(t_{0}\right) \wp_{k^{\prime} \mu 1} \wp_{k^{\prime} \mu 2}}
$$

These weights $w_{k}$ allow one to express the final density matrix $\underline{D}(x, t)$, using the final quantum state matrices $\underline{Q}_{t}(x, t)$ and Eq. (3.3). For each $\underline{Q}_{k}$, there is a normalization factor $\mathcal{N}_{k}$ equal to a hypothetical joint probability which we would have computed if $V$ were infinite. In general, that factor $\mathcal{N}_{k}$ is not equal to the product $\varphi_{k \mu 1} \varphi_{k \mu 2}$. This makes expressions of the density matrix $\underline{D}(x, t)$ a little more complicated.

A case of interest is the case where a set of values $\mu_{1} \mu_{2}$ for the outcomes of the measurements is given a probability zero by quantum theory and a non-zero value by Eq. (4.16). That set of values may be taken by $\mu_{1}$ and $\mu_{2}$ in measurements closely spaced in time. The approximation $\lambda_{m} \tau_{m}=\infty$ makes no sense in this case and the operators $\tilde{\Pi}_{\mu 1}$ and $\tilde{\Pi}_{\mu 2}$ in Eqs. (D.2) and (D.3) have to be replaced by $\Lambda_{\mu 1}$ and $\Lambda_{\mu 2}$ as defined by Eq. (C.3). The expression of the final $\tilde{Q}(x, t)$ is more complicated but $\mathcal{N}$ is not zero and no divergence is encountered. 


\section{FIGURE CAPTIONS}

Fig. 1. Case of two points of measurement $m_{1}$ and $m_{2}$ in the light cones of one another. The transition zone generated at $m_{1}$ has time to cover the distance between them at the velocity $V>c$ before the measurement at $m_{2}$ is performed.

Fig. 2. Case of two points of measurement $m_{1}$ and $m_{2}$ outside of the light cones of one another but such that the transition zone generated at $m_{1}$ has still time to propagate at the velocity $V>c$ across the distance separating $m_{1}$ and $m_{2}$ in the time interval.

Fig. 3. Case of two measurements, $m_{1}$ and $m_{2}$ closely spaced in time. Neither light nor a transition zone propagating at the velocity $V>c$ has enough time to travel from $m_{1}$ to $m_{2}$.

Fig. 4. Space time display of three points of measurement such that communication faster than light is possible in principle: $m_{1}$ and $m_{2}$ are closely spaced in time, $m_{3}$ is well spaced in time with respect to $m_{1}$ and $m_{2}$. 

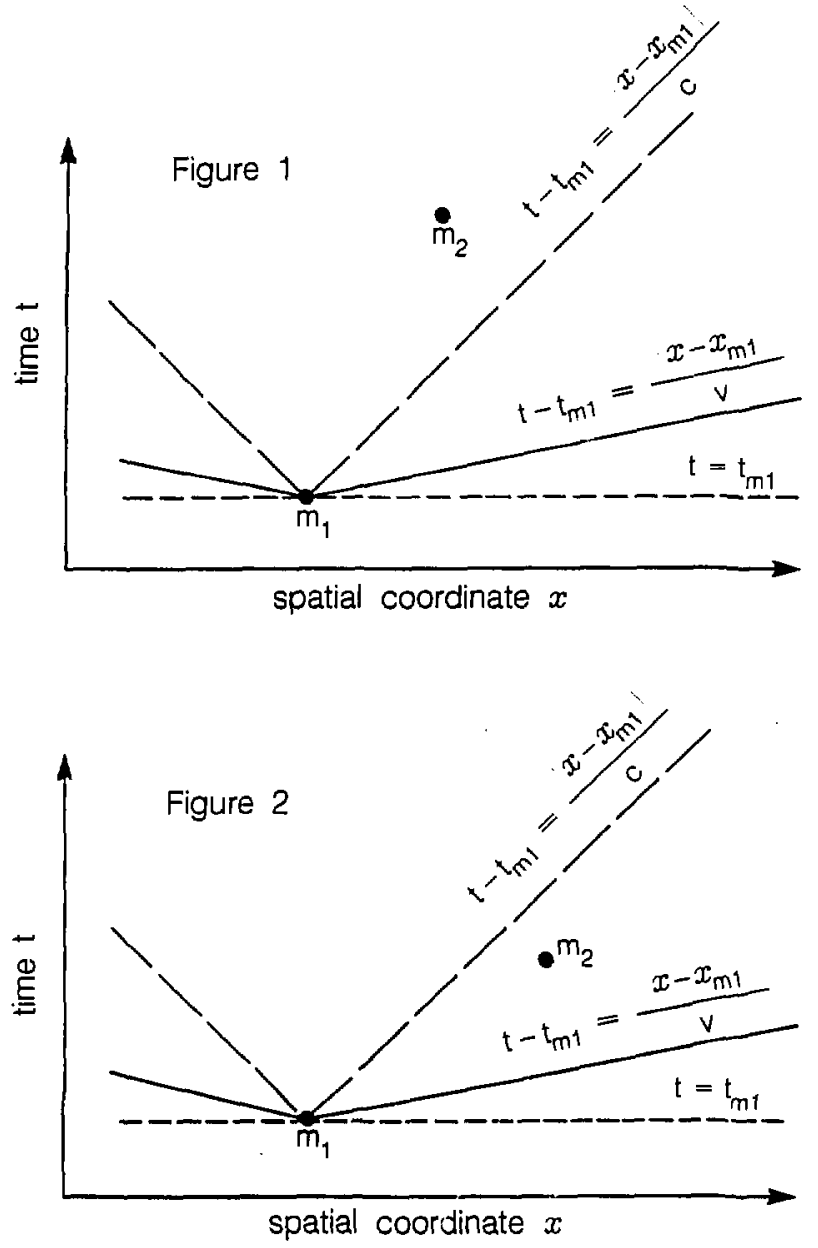

XBL 876-10195 

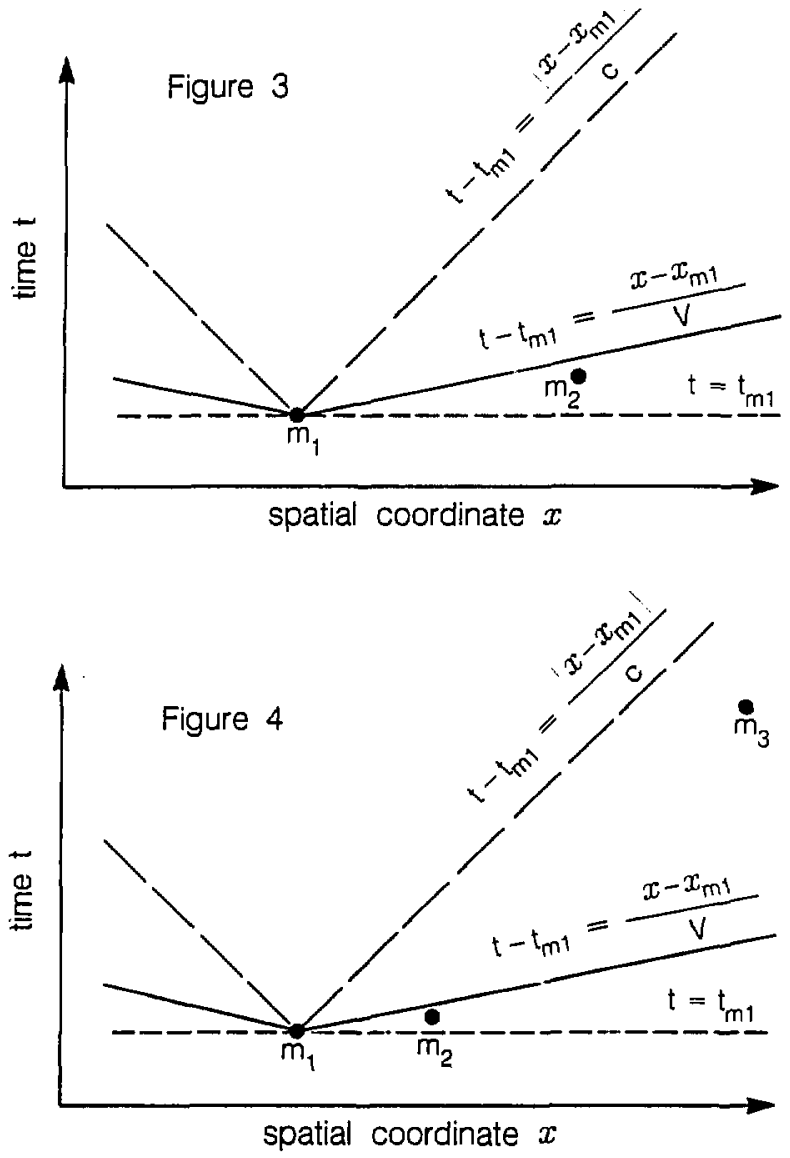

XBL 876.10194 